\title{
Estimating high precision hole diameters of aerospace alloys using artificial intelligence systems: a comparative analysis of different techniques
}

\author{
P. R. Aguiar ${ }^{1}$ R. B. Da Silva ${ }^{2}$ T. M. Gerônimo ${ }^{1}$ M. N. Franchin ${ }^{1}$ E. C. Bianchi ${ }^{1}$
}

Received: 1 November 2015 / Accepted: 10 March 2016 / Published online: 19 March 2016

(C) The Brazilian Society of Mechanical Sciences and Engineering 2016

\begin{abstract}
Monitoring metal removal in machining processes has proved to be essential for companies seeking a high level of excellence in the quality of their products and processes, contributing to improved resource allocation and reduced wastage due to nonconforming parts. Multisensory approaches have been employed to monitor these processes, aiming to use signals to train artificial intelligence systems to perform the task of indicating nonconformities in the tools or the product being manufactured. In this study, three artificial intelligence systems were used to estimate diameter of holes produced in sandwich plates-Ti6Al4V alloy was mounted in AA 2024-T3 alloy-and cutting conditions were selected to simulate a common aircraft fuselage manufacturing process. A multilayer perceptron artificial neural network (MLP ANN), an adaptive neurofuzzy inference system (ANFIS) and a radial basis function
\end{abstract}

Technical Editor: Márcio Bacci da Silva.

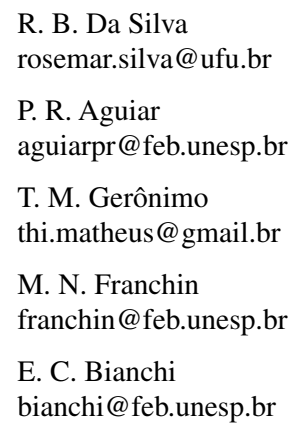

R. B. Da Silva

rosemar.silva@ufu.br

P. R. Aguiar

aguiarpr@feb.unesp.br

T. M. Gerônimo

thi.matheus@gmail.br

M. N. Franchin

franchin@feb.unesp.br

E. C. Bianchi

bianchi@feb.unesp.br

1 Faculty of Engineering, UNESP, Paulista State University, Av. Eng. Luiz Edmundo C. Coube 14-01, Bauru, SP 17033-360, Brazil

2 School of Mechanical Engineering, UFU, Federal University of Uberlandia, Av. Joao Naves de Avila, 2121, Bloco 1-M, Uberlandia, MG 38400-902, Brazil
(RBF) neural network were trained to estimate the diameter of machined holes. The multisensory approach includes an acoustic emission sensor, accelerometer, dynamometer and an electric power sensor. The optimum configuration for each artificial intelligence system was determined based on algorithms designed to examine the influence of each system's signals and specific parameters on the final result of the estimate. The results indicated the MLP ANN was more robust in withstanding data variation. The ANFIS system and RBF network showed markedly varying results in response to variations in the obtained data during training, suggesting these systems should always be trained with the dataset presented in the same order. A satisfactory response between the multisensory approach and MLP network was observed. The vertical component of force, along the $z$ axis, was the only parameter able to present valid results for all the artificial intelligence systems analysed.

Keywords Artificial intelligence systems · Drilling process monitoring - ANFIS - RBF · ANN $\cdot$ Hole diameter · Experimental trials $\cdot$ Cutting forces $\cdot$ Aerospace alloys

\section{Introduction}

The control of industrial metal removal processes is usually of great economic importance because of the efforts to reduce raw materials, water use and electrical power consumption as well as labor waste. Indirect manufacturing operations, such as dimensional quality control, generate indirect costs that can be avoided or reduced using control systems [1]. The next step in manufacturing process monitoring is intelligent manufacturing systems (IMS), which have been studied since the 1980s through the application of artificial neural networks (ANN) [2]. 
Automated manufacturing systems have gained ground and are applied in machining centers or individually in $\mathrm{CNC}$ machines. Automation techniques used in manufacturing are applied to the processing, assembly, inspection and handling of products.

In inspection, various computer-aided quality control methods have been employed in industry. These methods include control by visual inspection through the application of intelligent systems for detecting defects [3], analysis of acoustic emission and vibration signals to determine grinding wheel wear, and estimation of surface roughness in grinding processes [4].

Nearly $90 \%$ of the all engineering components are subjected to some operation of machining during manufacture. Drilling is one of the most common machining processes employed in industrial manufacturing and occupies a prominent position in the metal working industry, being responsible for removing $25 \%$ of all the removed material in industrial processes, especially in automotive industry. Most of round holes are manufactured with twist drills and need to be inspected. Quality inspection is usually based on precision of diameter and form errors (circularity, cilindricity and roughness) according to the design tolerances required. Drill wear is generally monitored using direct methods such as optical devices. To enable such inspection, the process must be stopped and both drill and machined part should be carried out to a specific gate in line production what increases production time and costs [5]. In addition, drill point is hidden inside the hole during operation and chip evacuation is hampered when drilling blind holes, what are unfavorable conditions compared to turning and milling operations.

When a cutting edge and/or margin of drill wear out, the cutting phenomena of drilling process becomes complex, and as wear progresses, force and power required to keep machining is increased. During machining component forces are exerted on the outer cutting lips and cutting tools are subjected to an extremely severe rubbing process. So, the mechanical energy necessary for the drilling process is transformed into heat, thereby leading to severe thermal/ frictional conditions at the tool-chip interface. As a result of this, high cutting temperatures are generated on this interface and can lead to accelerate wear rate, chipping and ultimately failure. If drill is not changed before its degradation, hole's quality will be compromised. Therefore, monitoring of machine power or forces and torque during machining are an important aspect to be considered and has attracted great interest from metal working industry. But the major challenge in drilling monitoring is the diagnostic capability of the systems in the real-time correct identification of the drilling tool condition, i.e., to achieve the correct correlation between drill wear and signals being acquired in real time monitoring.
It has been reported in the literature that the first work using artificial neural networks (ANN) in monitoring machining processes was carried out by Rangwala and Dornfeld [6] at the end of the 1980s. These authors developed a back-propagation network to classify sharp and worn tools in turning operation. In drilling operation, the artificial neural networks have been employed in monitoring of drill wear with aid of sensors that can acquire many signals. The various types of signals employed include those produced during machining and obtained by dynamometer loads [7], electrical current obtained by the application of Hall-effect sensors on electric motors [8], vibrations [9] and also a combination of these and other sensors such as accelerometers and acoustic emission sensors [10]. The status of the wear is analysed with base on input variables such as cutting speed, feed rate, drill diameter, drill geometry among others.

The use of artificial intelligence systems has filled a gap in obtaining mathematical models for complex systems. During this research, no mathematical models were found in scientific articles or books to analyse the drilling process dynamically to determine the final hole geometry.

In this context, this research aims to contribute to ratify the use of artificial intelligence systems in evaluating the end result of machining manufacturing processes. So, a comparative analysis of the performance of three artificial intelligence techniques to estimate the diameter of holes machined by high-precision drilling in two metallic materials widely employed in the aircraft industry. This investigation also contributes to extend the work of Cruz et al. [5], who employed multilayer perceptron artificial neural networks (MLP ANNs) to analyse the relationship between the signals obtained in monitoring the drilling process and the hole diameters obtained in the process.

\section{Literature review}

Machining represent a large portion of the manufacturing processes employed in industry today, and it is estimated that about $70 \%$ of manufactured products are produced by this type of process, $10 \%$ of which correspond to drilling processes [11].

Whatever the field of activity may be, all manufacturers share common goals such as maximization of productivity allied to minimization of direct operating costs and indirect manufacturing or overhead costs, which are widely applied in setting goals and objectives for manufacturing processes. Overhead costs include: depreciation of machinery and equipment used in production, supervisor salaries and labor costs, energy consumed by manufacturing processes (electrical, pneumatic, etc.), environmental impacts and worker injuries caused by the use of cutting fluids, and quality 
control costs. The latter contributes to the costs of labor and to the acquisition and maintenance-including the calibration and repair-of measuring instruments and the costs of operators, as well as the costs involved in ensuring suitable conditions for taking measurements. Like the movement of materials, their storage time and the time spent in waiting for the next operation, the activity of inspection is considered a transaction that does not add value to the final product [1].

To reduce the costs of inspection activities, industry uses control systems to correct operating parameters by monitoring signals to keep the product's characteristics within acceptable limits. Monostori et al. [12] have described the control, monitoring and diagnostics of industrial processes as fields that overlap in terms of the techniques that are used, such as signal processing to detect abnormal conditions during the operation.

Manufacturing processes can be monitored to verify tool wear and also to detect cutting tool's breakage during the process by analysing signals to measure and estimate the process variables. This approach is known as tool condition monitoring. Other approaches consist of determining the surface integrity or dimensional accuracy of the workpiece. Liang et al. [13] reported the state of the art in the control and monitoring of industrial processes, and relate the monitored characteristics to the types of signals used in various applications.

Control over a process depends on the knowledge of a model that relates the parameters and variables of this process to the expected results of an architecture, which will define the adjustments needed to meet the desired characteristics. The model of a process can be obtained by two strategies: mathematical formulation by differential equations or, if a large amount of data is available, an artificial intelligence system can be used for the approximation [14]. Mathematical models are developed when one is knowledgeable about the relationship between the components involved. However, high complexity allied to nonlinearities in the tool and workpiece behavior during the process hinders the application of such models and the determination of an analytical solution. Furthermore, the simulation of complex models requires high computational performance.

To be classified as intelligent, a system must have characteristics that are related to human intelligence, such as memorization, the use of knowledge (data) in problem solving, and the ability to learn from experience [15]. Expert systems and artificial neural networks (ANN) are two artificial intelligence tools that can be used in process control. According to Wilamowski and Irwin [16], the knowledge contained in an ANN is not available in an understandable way, while an expert system can provide information about how and why a particular solution was obtained.
According to Huang and Zhang [2], computer integrated manufacturing is always under continuous improvement, so the application of intelligent systems is the next step in production control, as indicated by the considerable increase in the number of scientific articles published since the 1980s. The authors present a survey of ANN applications in manufacturing to support activities involving product design, process planning, production scheduling, modeling and control, monitoring and diagnostics, quality control, parts classification and robotics.

The term soft computing, which was proposed by Zadeh [17], reflects the need to consider the vagueness and uncertainty that are part of the human decision-making process in the analysis of real problems for intelligent embedded systems. The reason for this is that humans make better decisions based on imprecise information than on meticulously detailed information, e.g., when parking a car, the task of parking was performed based only on the driver's perception of the dimensions of the parking space.

Soft computing consists of the fusion of fuzzy logic with artificial neural networks and evolutionary computation, resulting in an intelligent system that is able to combine the characteristics of each component, such as imprecision, learning and uncertainty, respectively [18].

According to Dote and Ovaska [19], industrial applications of soft computing include the aviation sector, communication systems, home appliances, power systems, manufacturing automation and robotics, power electronics and motion control, and processes and transport engineering. Intelligent systems are therefore very effective when applied to real problems, when the traditional approach is difficult to apply due to the need for better models.

Artificial neural networks are very useful when working with reactive quality control, which, according to Huang and Zhang [2], includes sampling planning, batch acceptance analysis and rework analysis.

Chinnam et al. [20] proposed an online quality control method based on process control during the manufacturing operation, which involves the use of a multilayer perceptron artificial neural network (MLP ANN) with three hidden layers to build a relationship between outputs and process parameters.

In the monitoring of machining operations, knowledge of the mathematical model of the process is used for control, based on the physics of their interactions. This model is usually approximated by differential equations that can be applied to simulation software by the finite element method, allowing the behavior of the process to be analysed, albeit with great computational effort. However, due to the complexity of the tool/workpiece interaction understanding, accurate mathematical models are difficult to be obtained. Soft computing methods such as ANN and genetic algorithms can be used to model the process based 


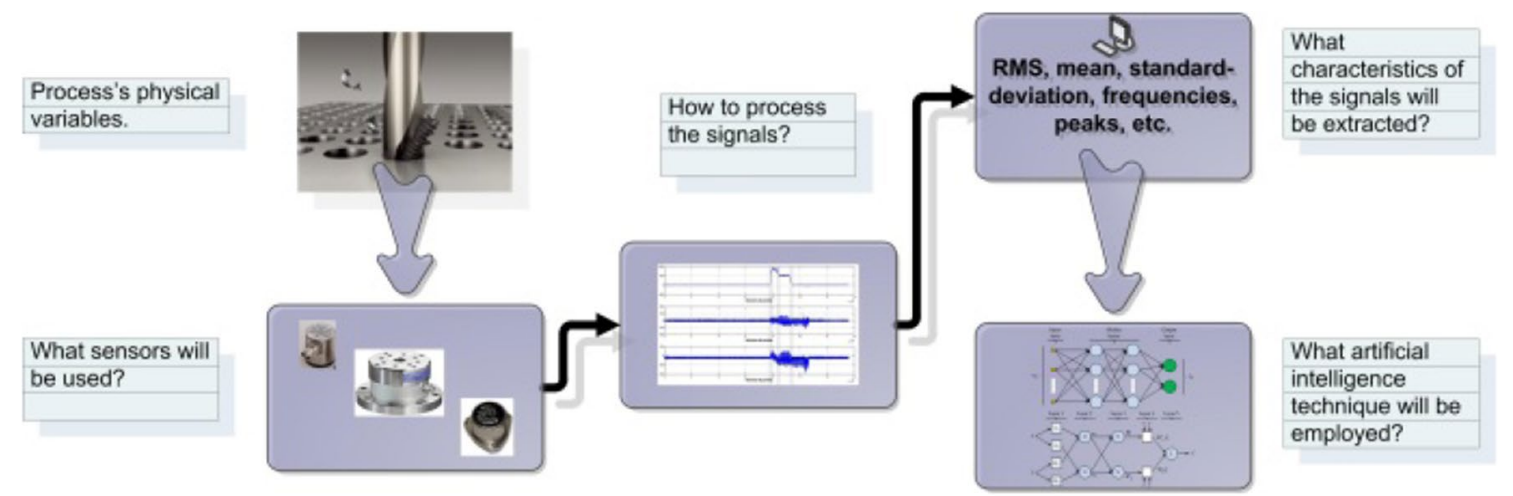

Fig. 1 Generic method for developing an intelligent monitoring system for machining processes (adapted from Abellan-Nebot and Subirón [23])

on collected data. The advantage of using this kind of solution lies in its ability to relate outputs to inputs, despite inaccurate, missing or uncertain data [14].

Abu-Mahfouz [9] proposed a tool condition monitoring system to enable more efficient and economical use of a twist drill during dry machining of an AISI 1040 steel grade. The author employed an accelerometer to capture the flange vibrations, disregarding the input and output transients of the drill in the workpiece. An MLP ANN was trained using the feed, the tool's rotation and four statistical parameters resulting from processing the acceleration signal to detect five types of drill wear. The MLP network was able to detect wear in all the training sets and achieved about $80 \%$ success rate in classifying the type of wear, demonstrating the capacity of the acceleration signal to monitor manufacturing processes.

Hermann [21] has investigated the application of neural network based sensor fusion in monitoring of drill wear and failure and trained an MLP ANN by the backpropagation method to identify both problems, based on acoustic emission signals, vibration and feed force. Online monitoring was carried out with a conventional milling machine and the accurate recognition of drill wear was analysed in relation to the input signals presented to the MLP network. The author reported that the best results in terms of detect drill wear were obtained by combining the mean values of acoustic emission and feed force signals.

Sensors and sensing techniques, control strategies and open-source software available for monitoring manufacturing processes were discussed by Liang et al. [13]. Advances in these fields are expected to involve the use of data to control chip and burr formation. Process information will be collected more comprehensively with more sensors to monitor several variables.

Bustillo et al. [22] carried out a study using a virtual sensor for online fault detection of multi-tooth tools during milling operation of engine crankshafts based on a Bayesian model and used the electrical power consumption and the machining time as output variables. These authors reported that a 26 workpiece interval before the workpiece that is machined in real time is necessary for fault detection, against 40-70 workpiece interval observed in previous works, as well as a measured accuracy of $98 \%$.

An approach for controlling machining processes consists of tool condition monitoring. This approach involves collecting data, generally using multiple sensors to determine the wear level, indicating the most economical moment for changing the tool. Kandilli et al. [10] monitored drilling and milling processes in real time using force sensors ( $x, y$ and $z$ ), acceleration, current, and acoustic emission installed in a 4-axis CNC machining center. The mean, standard deviation and RMS of the collected signals were presented to an MLP ANN to identify wear.

A review of artificial intelligence-based monitoring systems for machining processes is presented in AbellanNebot and Subirón [23]. Their survey considered the different types of sensors used, the signal processing techniques, the characteristics of the signals, and the methods used for their extraction, the design of experiments to use the least possible amount of data, and lastly, the characteristics of the artificial intelligence techniques applied, to relate them to process outputs. Figure 1 shows their proposal for the implementation of intelligent monitoring of a manufacturing process.

Bustillo and Correa [24] employed a Bayesian network to predict the surface roughness of holes drilled in steel workpieces. Their results were divided into classes of roughness, and the network accurately classified $86 \%$ of the estimates.

In other research, Ferreiro et al. [25] employed data mining techniques to obtain a reliable model to detect the generation of burr in holes of an aeronautical aluminum alloy, Al 7075-T6 grade, after drilling operation with carbide drills. Since data mining is a process employed to describe knowledge discovery and to search for significant relationships, such as patterns, association, and changes among 
variables in databases, in this research the authors aimed to study the sensitive of different signals to the burr detection, to treat and to use them to develop on-line monitoring system, thereby approximating the optimal model to the industrial real applications. In regard of feature selection strategy, the authors reported that is important to find the most influential variables that can improve the model in term of their accuracy, validity, reliability and stability. Torque of the spindle, electrical power consumed and axial force were the selected signals because of some advantages presented by these authors such as: (1) the relative simplicity of the acquisition method and because no elements are added to the workpiece, resulting in easy implementation, since there are not external sensors; (2) the elimination of unproductive operations by the reduction in the number of inspections and operations to remove burr and consequently reduction in costs.

Grzenda et al. [26] carried out a research that combines genetic algorithms and multilayer perceptrons (MLP) to optimize deep drilling operation in terms of improving the detection of holes burr under different cutting conditions. Their research was divided into three phases (new strategy to evaluate and complete the set of available measurements, a bag of MLP to model the impact of deep drilling settings on borehole roughness and a model supplied with the hole dimensions, coolant delivery technique (traditional and MQL one) and expected axial force) to finally develop a 3D surface of a surface roughness of a hole as a function of drilling process parameters previously mentioned. The authors reported that the error rate of prediction performed for the data sets, which are imputed using a strategy, can provide the basis for a fitness function, i.e., the higher the accuracy of the roughness prediction performed on the imputed data set, the better the imputation strategy used to fill in the missing values. Seven input variables were employed in the experimental trials: tool type, tool diameter, hole length, feed rate, cutting speed, coolant delivery technique and axial cutting force (parameter that the authors considered as one of the main inputs to model the drilling process). The authors observed that the MLP approach generated reasonable results, since the algorithm using a combination of evolutionary approach and MLP networks was used to select the imputation strategy and to increase the number of measurements available for smooth roughness modelling. These authors also found that the lowest roughness values provided by the model (3D surface response) was obtained after machining a hole with $50 \mathrm{~mm}$ length with a drill of $10 \mathrm{~mm}$ diameter (designed as a drill 1), feed rate in the range of $0.22-0.24 \mathrm{~mm} / \mathrm{rev}$, cutting speed in the range of $90-120 \mathrm{~m} / \mathrm{min}$ under the MQL technique.

Despite the good results obtained by applying artificial intelligence systems to monitor machining processes, studies focused on estimating the final result of drilling process are still few. Into this context, this paper gives the contribution to machining field presenting the predicted results of hole diameters performed in sandwich plates of Ti6Al4V alloy/AA 2024-T3 alloy using three artificial intelligence systems. Experimental machining trials were also performed.

\section{Materials and methods}

This section discusses the implementation of artificial intelligence systems, and the methodology used to define their configurations. All the information about the tests and signal acquisition and processing was obtained from Cruz et al. [5].

\subsection{Experimental trials and measurements}

The drilling process was carried out in a Zocca U-30 universal milling machine that is driven by a $6 \mathrm{CV}$ motor drive, a variable spindle speed from 45 to $2800 \mathrm{rpm}$ and a variable feed rate ranging from 4 to $250 \mathrm{~mm} / \mathrm{min}$, with axis position control via HMI. The milling machine also has electronic rulers with micrometric precision in each axis, which was used to ensure that the coordinates of the holes in the Zocca workpiece specimen would be standardized [5].

The data used for training artificial intelligence systems consist of the results of monitoring a drilling process. Nine identical uncoated cemented carbide helical drills with $4.95 \mathrm{~mm}$ of diameter were employed in all experimental trials. They are recommended for machining alloys from aviation industry. The tools were manufactured by OSG Sulamericana de Ferramentas Ltda (Brazil). All the experimental trials were carried out without cutting fluid (dry condition). Each of the nine carbide drills was identified with a serial number. One fresh drill was used to perform a total of 162 holes. This number was selected based on previous machining trials that resulted in significant tool wear, and consequently allowing variation in the acquired output signals.

The outer diameter of each drill was measured with a profile projector; and the average of five measurements was stored for use in training the artificial intelligence systems. It is extremely important to know the numerical value of the diameter of the drill so that, together with the tool rotation value, it is possible to determine the cutting speed, which is essential in machining.

The drilling operation was performed in a sandwich plate of commercially available alpha-beta titanium-base, Ti-6Al-4V, alloy bonded with interface sealing solution to a sheet of aluminum-base, AA2024-T3, alloy. Dimensions 
Fig. 2 Diagram illustrating the mount position of each sensor and the signal acquisition with the reference system indicating the direction of cutting forces (Cruz et al. [5])

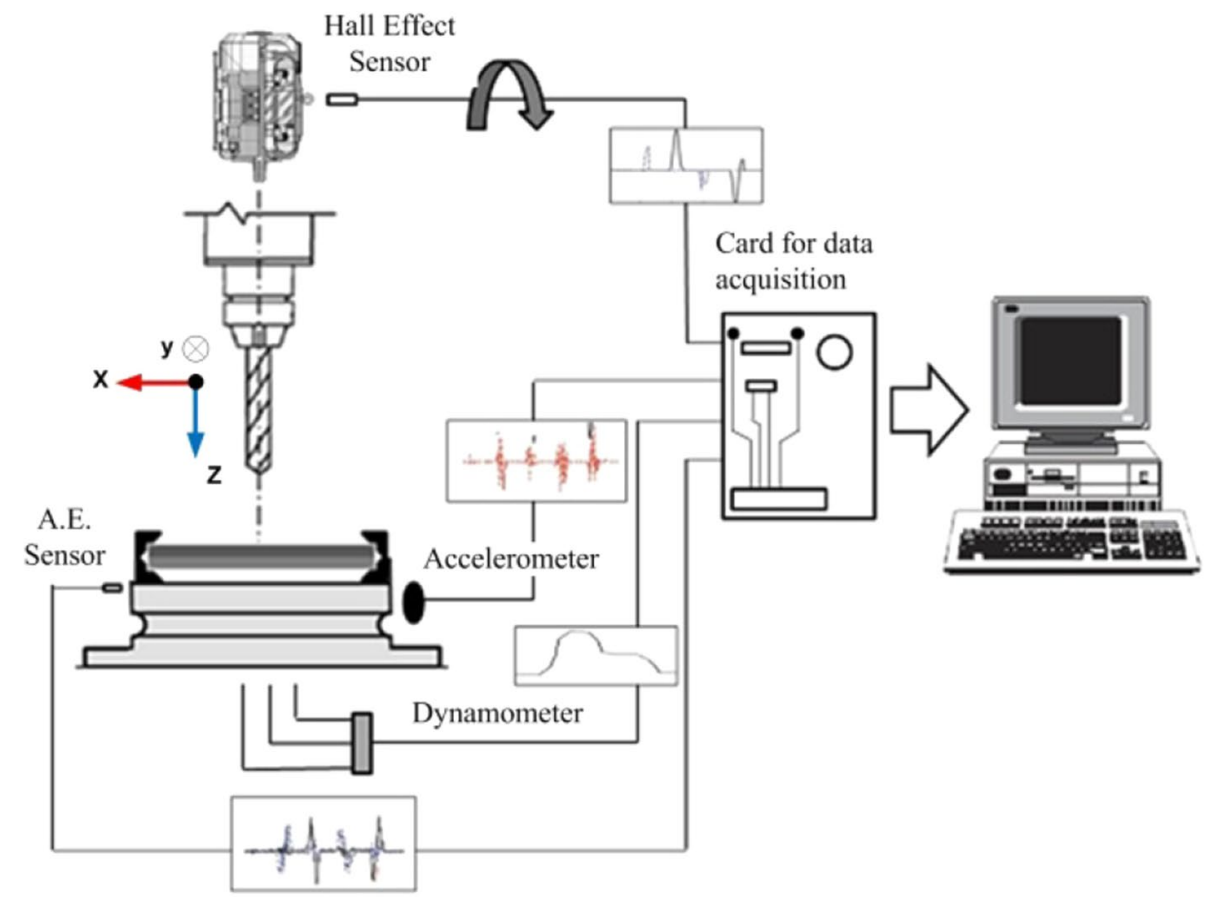

of workpiece are $150 \mathrm{~mm}$ length $\times 50 \mathrm{~mm}$ width $\times 3 \mathrm{~mm}$ height. A total of 54 workpiece specimens were machined to produce holes under different sets of machining conditions, i.e., six specimens in each of nine sets.

The cutting parameters were selected to represent various machining conditions used in the aviation industry. Thus, three values of cutting speed $\left(v_{\mathrm{c}}\right), 7.8,15.5$ and $31.1 \mathrm{~m} / \mathrm{min}$, and three values of feed rate $(f), 0.05,0.09$ and $0.125 \mathrm{~mm} / \mathrm{rev}$, were combined, which resulted in nine different sets of conditions.

Four sensors were installed to monitor the drilling process: an acoustic emission sensor, a 1D accelerometer, a Hall-effect sensor, and a 3D dynamometer. The reason for using these sensors is because they are the most used in monitoring of machining processes. The signals were transferred to a computer via a National Instruments NI PCI-6035E data acquisition board, 16-bit, with an acquisition rate of $2 \mathrm{kS} / \mathrm{s}$. Figure 3 illustrates the setup of the signal acquisition modules with the computer and the machine tool.

Each sensor captured a characteristic of the drilling process by measuring the following variables:

- The acoustic emission (AE) of the material, which consists of the propagation of transient elastic waves generated by the rapid release of sources of energy in it. The sensor was connected to a test bench used to fix the dynamometer to the workpiece specimen and to the machine tool table top to capture the acoustic emission generated by the deformation, shear and/or rupture of the machined material;
- The vibrations (acceleration-ACC) in the machine, which are due to the forces transmitted to a workpiece during machining, were measured with aid of accelerometer. Both AE and ACC signals were measured in the $x$ direction (Fig. 2) because such axis showed higher sensitivity;

- Hall-effect sensors were used to measure the current and voltage of the induction motor. Both signals were multiplied and filtered, resulting in a signal corresponding to the electrical power consumed;

- The component forces acting in machining process are related to the material's resistance, shear and yield, which determine the choice of the cutting parameters. A 3D dynamometer was installed to collect information about the forces in the $x y$ plane and the effective cutting force along the $z$ axis. A test bench was installed to ensure the rigidity of the workpiece specimen/sensor/ table top setup to mount this sensor (Fig. 2), which is fundamental for the performance of drilling;

- The collected signals were analysed by computational routines in MATLAB ${ }^{\circledR}$ to eliminate the portions before and after the cutting operation was performed when the tool was not in contact with the workpiece. The absolute average value of the region of each signal was calculated and used as an input for the ANN, in addition to the cutting speed and feed rate employed in the condition under analysis, as previously reported by Cruz et al. [5]. Examples of a typical ANN input data can be found in the works carried out by Cruz et al. [5] and Ferreiro et al. [25] and Grzenda et al. [26]. Feed rate and cutting speed were one of the input variables selected by 
Fig. 3 Division of the collected signals according to the machined material, where twofifths (yellow) correspond to the Ti6Al4V alloy and three-fifths (white) to the AA 2024-T3 alloy (Cruz et al. [5]) (color figure online)
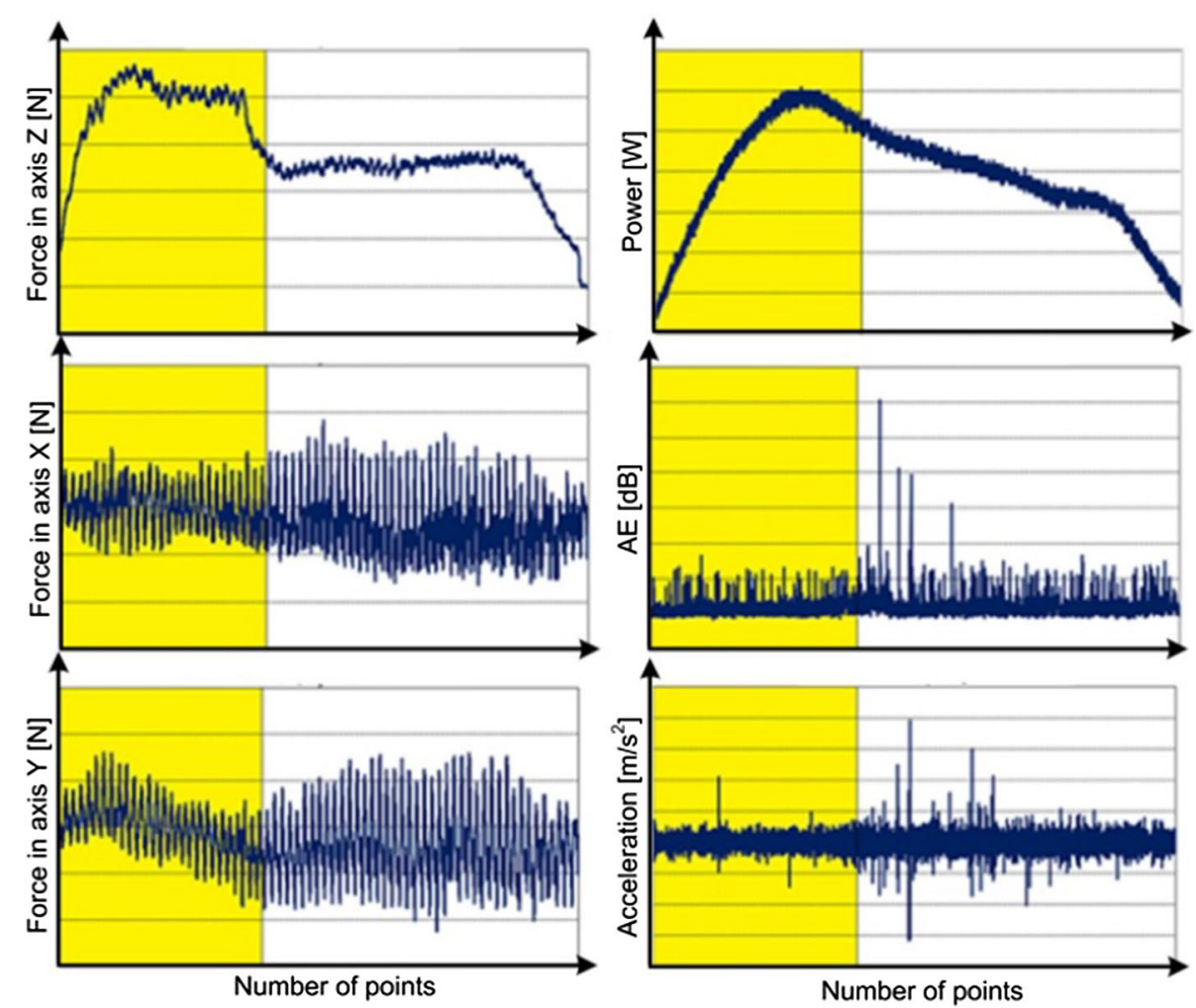

Grzenda et al. [26] when applying a soft computing system based on intelligent imputation strategies for roughness prediction in deep drilling of F114 (AISI 1045) steel grade with various operational parameters.

It worth mentioning that the neural network models acted as feature extraction tools, in which many input combinations further described were tested and analysed regarding the least error obtained. In addition, the early stopping technique was used in this work to avoid overfitting problem and improve generalization ability of the neural network models by monitoring error trend of training set, validation set and test set during training $\mathrm{Wu}$ and Liu [27].

In this work, the cross-validation procedure was applied to provide better generalization of neural network models. To perform the cross-validation procedure input data was partitioned into three sets: training, validation and test sets. To ensure a correct comparison of different types of neural networks the division of input data into training, validation and test sets were performed by independent part of code and the division result was stored. The partitioning of input data was performed randomly with a certain ratio of input entities to be stored as training set, validation set and test set $(0.6,0.2$ and 0.2 , respectively).

The vertical component of force along the $z$ axis (z-direction), labeled here as $\mathrm{Z}$ component force, was used to determine the beginning and end of the operation, because among all the signals collected, this was the one that best represented the two stages. The regions of inactivity were ignored, as it was the portion of the signal corresponding to the withdrawal of the drill bit from the machined hole.

Because of each workpiece material has different properties like mechanical resistance, for instance, the holes will present different dimensional deviations as well as the recorded signals will show different patterns during machining. Because the different physical properties and consequently different response in signals for Ti6Al4V and AA 2024-T3 alloys, the signals were divided into two portions: two-fifths to the Ti6Al4V alloy and three-fifths to the AA 2024-T3 alloy, as illustrated in Fig. 3. For these signals, the arithmetic mean of the absolute values of each signal was calculated to obtain an average value of each signal for each hole drilled in the workpiece specimens.

The holes diameters were measured with the aid of a Mahr $1087 \mathrm{~B}$ dial indicator with $\pm 0.005 \mathrm{~mm}$ precision. To preclude the influence of swarf and chips produced in the drilling process, the workpiece specimens were cleaned with compressed air and dusting cloths.

Due to the alloys' different properties, each one shows a distinct dimensional behavior differently. Thus, the hole diameter obtained was expected to present circularity deviations and was approximated by an ellipse indicating minimum and maximum dimensions. 
In this work the total data provided by experimental trials was randomly divided to obtain the ANNs estimation error in accordance with the following percentage: $60 \%$ used ANN training, $20 \%$ for validation and $20 \%$ for ANN test to confirm the performance of each neural network tested. Due to the adopted measuring procedure, four diameter values were recorded for each measured hole, representing the minimum and maximum hole diameters in each of the alloys. The records obtained were used to compose the training set of the artificial intelligence systems, in terms of the desired outputs.

\subsection{Configuration of artificial intelligence systems used for estimating the diameter of machined holes}

\subsubsection{MLP network}

In this study, an MLP ANN was used which was configured and implemented in MATLAB ${ }^{\circledR}$ using the ANN toolbox application. The MLP was configured using an algorithm proposed by Cruz et al. [5], which is aimed at varying the architecture of the neural network and two parameters, as illustrated in Fig. 4.
After obtaining the results, the MLP network configuration was chosen based on the classification of the mean error obtained in the estimate of the minimum and maximum diameters. This configuration was selected based on a previous analysis made of the network among the five best ones that provided the lowest mean error in terms of both minimum and maximum diameters.

After the classification, a new algorithm was developed to verify the influence of the average of each of the collected signals on the network's performance. The influence of the combination of two signals was also considered. This procedure was adopted to obtain a process monitoring strategy involving the use of the fewest possible sensors, thus reducing implementation costs and interference in the manufacturing process. The new algorithm is illustrated in the flowchart in Fig. 5.

\subsubsection{ANFIS systems}

The configuration of the ANFIS system is defined based on the determination of a fuzzy inference system (FIS) with its components, as shown in the following section, and the subsequent adjustment of the pertinence functions, rules, and weights.
Fig. 4 Flowchart representing the algorithm for testing the MLP network architecture and parameters, proposed by $\mathrm{Cruz}$ et al. [5]

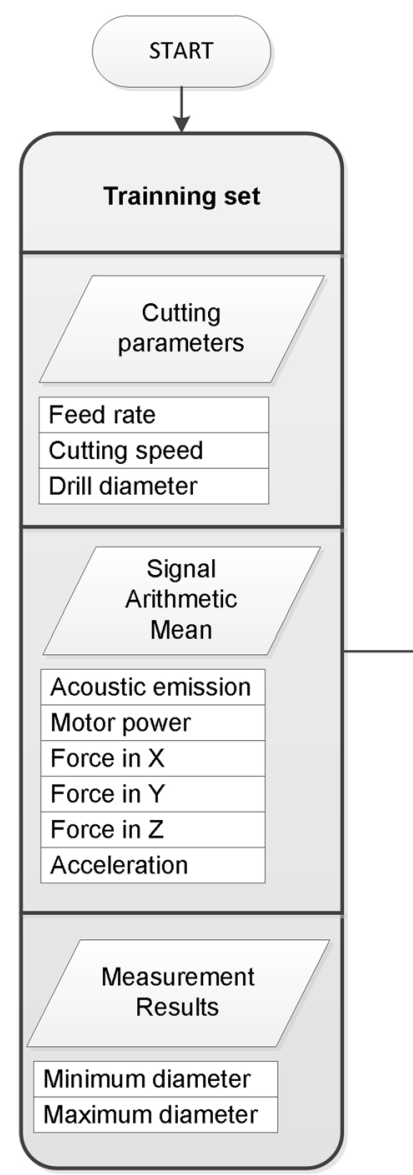


Fig. 5 Flowchart representing the algorithm for testing the signals
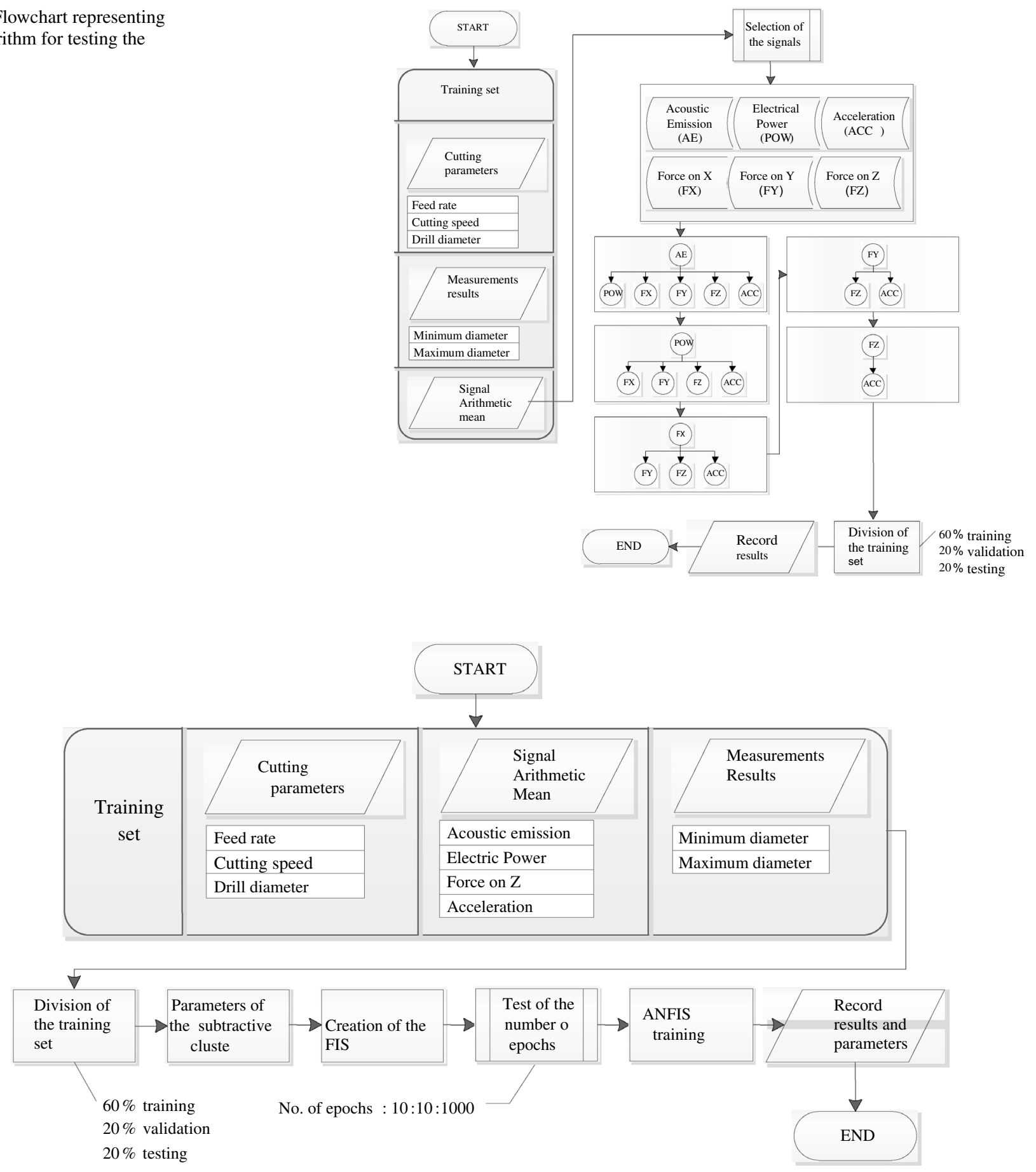

Fig. 6 Flowchart representing the algorithm used for ascertaining the influence of the number of training epochs

The tests include the evaluation of the influence of the cluster centers on the definition of the FIS rules, determined by trial and error, and of the influence of the combined input signals.

Like in the implementation of the MLP network, the ANFIS system available in MATLAB's Fuzzy toolbox was used. Because the system can support the generation of only one output, four ANFIS systems were developed, two for each alloy of the workpiece specimen. The results were analysed using the mean squared error generated at the output of the ANFIS. Another limitation inherent to MATLAB ${ }^{\circledR}$ is that a maximum of four simultaneous input signals can be used. 
Fig. 7 Flowchart representing the algorithm developed to test the influence of the signals and the support of the Gaussian functions
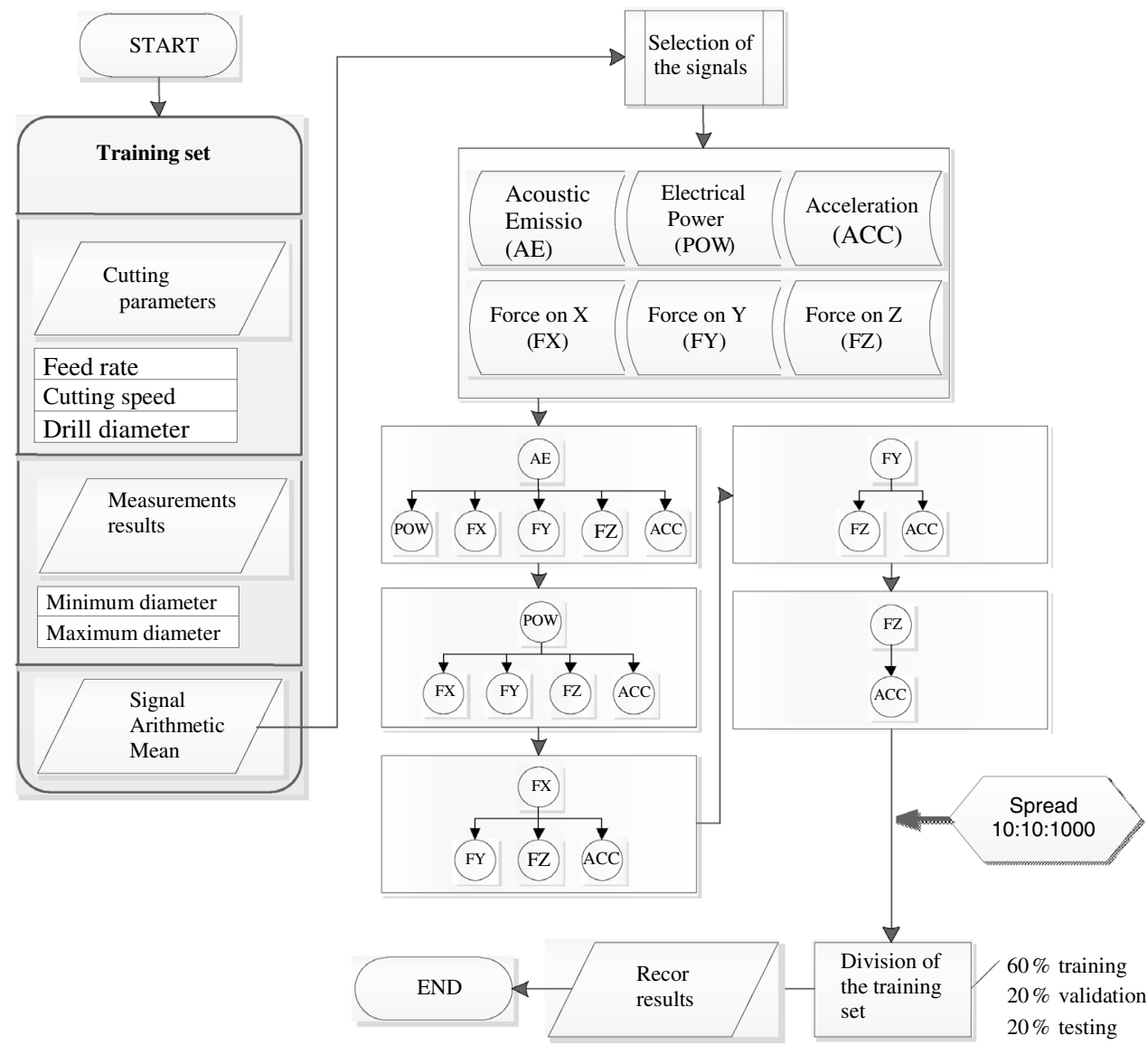

An algorithm was developed to analyse the influence of the number of training epochs (Fig. 6). To test the influence of input signals, the MATLAB ${ }^{\circledR}$ algorithm was used with a method of combination of inputs, known as a sequential method, which is similar to the method developed for training the MLP network. Only the signals of acoustic emission, power, component of the machining force in the $Z$ direction, and acceleration were considered for this test.

\subsubsection{RBF network}

The tests conducted on the RBF network consisted of analysing the influence of the applied signals and scattering parameter (spread), which means the support, radius or standard deviation of a Gaussian function.

Due to reduced computational effort involved, which is justified by the need to check only one parameter, the RBF test algorithm included the signal tests simultaneously with the spread test. Thus, for each signal or combination of signals, the spread was varied from 10 to 1000 in steps of 10 .

Like the signal tests performed on the MLP and ANFIS networks, the sequential combination occurred in sets of two combined signals or an individual signal. The corresponding algorithm is represented in the flowchart in Fig. 7.

\subsection{Network evaluation criteria}

Three evaluation criteria were defined to analyse the results obtained in the estimation of the diameter of the networks:

- Mean error Calculation of the average of the absolute and relative mean errors obtained by estimating each pair of diameters. Equation (1) shows the calculation performed for a total number of samples " $n$ ":

$$
E=\frac{1}{n} \sum_{i=1}^{n}\left|D_{\text {measured }_{i}}-D_{\text {estimated }_{i}}\right|
$$

- Quality control Analysis of false positives or false negatives, leading to undue acceptance or rejection of the drilling results.

- Tolerances Error classification (EC) based on allowed dimensional deviations, which, according to the Brazilian NBR 6158 standard [28] are necessary for a precision drilling process (up to $\pm 48 \mu \mathrm{m}$ ), depending on the precision of the measuring instrument used $( \pm 5 \mu \mathrm{m})$, 
Table 1 Experimental results after drilling the Ti6Al4V alloy with different cutting conditions for different cutting conditions (hole number 160)

\begin{tabular}{|c|c|c|c|c|c|c|}
\hline \multicolumn{2}{|c|}{ Cutting conditions (input) } & \multicolumn{5}{|c|}{ Output parameter } \\
\hline $\begin{array}{l}\text { Cutting speed } \\
(\mathrm{m} / \mathrm{min})\end{array}$ & $\begin{array}{l}\text { Feed rate } \\
(\mathrm{mm} / \mathrm{rev})\end{array}$ & $\begin{array}{l}\text { Hole diameter } \\
(\mathrm{mm})\end{array}$ & $\begin{array}{l}\text { Acoustic emission } \\
\text { AE signal (V) }\end{array}$ & $\begin{array}{l}\text { Acceleration or } \\
\text { vibration ACC } \\
\text { signal }\left(\mathrm{m} / \mathrm{s}^{2}\right)\end{array}$ & $\begin{array}{l}\text { Electrical power } \\
\text { POW (W) }\end{array}$ & $\begin{array}{l}\text { Z component force } \\
(\mathrm{FZ})(\mathrm{N})\end{array}$ \\
\hline 7.8 & 0.05 & 4.97 & 0.01 & -9.16 & 29.62 & 30.61 \\
\hline 15.5 & & 4.97 & 0.02 & 13.92 & 24.72 & 81.33 \\
\hline 31.1 & & 4.97 & 0.02 & 27.45 & 6.25 & 144.29 \\
\hline 7.8 & 0.09 & 4.96 & 0.02 & -4.93 & 15.65 & 24.25 \\
\hline 15.5 & & 4.98 & 0.03 & 20.51 & 20.03 & 47.3 \\
\hline 31.1 & & 4.96 & 0.03 & 18.34 & 20.77 & 94.28 \\
\hline 7.8 & 0.125 & 5.02 & 0.06 & -6.7 & 59.73 & 21.25 \\
\hline 15.5 & & 4.98 & 0.08 & 12.64 & 30.14 & 29.24 \\
\hline 31.1 & & 4.98 & 0.08 & 18.56 & 14.07 & 52.45 \\
\hline
\end{tabular}

Table 2 Experimental results after drilling the AA 2024-T3 alloy with different cutting conditions for different cutting conditions (hole number 160)

\begin{tabular}{|c|c|c|c|c|c|c|}
\hline \multicolumn{2}{|c|}{ Cutting conditions (input) } & \multicolumn{5}{|c|}{ Output parameter } \\
\hline $\begin{array}{l}\text { Cutting speed } \\
(\mathrm{m} / \mathrm{min})\end{array}$ & $\begin{array}{l}\text { Feed rate } \\
(\mathrm{mm} / \mathrm{rev})\end{array}$ & $\begin{array}{l}\text { Hole diameter } \\
(\mathrm{mm})\end{array}$ & $\begin{array}{l}\text { Acoustic emission } \\
\text { AE signal (V) }\end{array}$ & $\begin{array}{l}\text { Acceleration or } \\
\text { vibration ACC } \\
\text { signal }\left(\mathrm{m} / \mathrm{s}^{2}\right)\end{array}$ & $\begin{array}{l}\text { Electrical power } \\
\text { POW (W) }\end{array}$ & $\begin{array}{l}\text { Z component force } \\
(\mathrm{FZ})(\mathrm{N})\end{array}$ \\
\hline 7.8 & 0.05 & 5.00 & 0.01 & -8.4 & 29.16 & 17.47 \\
\hline 15.5 & & 5.00 & 0.08 & 4.38 & 39.74 & 61.57 \\
\hline 31.1 & & 5.00 & 0.09 & 29 & 47.96 & 127.69 \\
\hline 7.8 & 0.09 & 4.98 & 0.03 & -4.68 & 11.31 & 11.98 \\
\hline 15.5 & & 5.02 & 0.04 & 21.96 & 31.26 & 27.86 \\
\hline 31.1 & & 4.98 & 0.11 & 17.97 & 60.48 & 66.57 \\
\hline 7.8 & 0.125 & 5.07 & 0.07 & 3.57 & 57.4 & 8.47 \\
\hline 15.5 & & 5.02 & 0.21 & 23.42 & 82.97 & 19.91 \\
\hline 31.1 & & 5.02 & 0.19 & 18.44 & 100.09 & 38.02 \\
\hline
\end{tabular}

and depending on the tolerance generally used in the industry $( \pm 12 \mu \mathrm{m})$.

\section{Results and discussion}

The methodology adopted to simulate the networks involved three different combinations of sets of input signals for each type of network, i.e., with all the collected signals, with a single input signal, and with specific combinations of input signals.

Due to the large number of results generated after machining each alloy, the hole number 160 was chosen because it was closer to the stipulated criterion to halt the drilling test (162 holes-condition that drill wear was significantly high), as mentioned previously in materials and methods section. The experimental results obtained for all the output parameters [diameter, the acoustic emission (AE), acceleration (ACC) and electrical power (POW) signals, as well as, the $\mathrm{Z}$ component force (FZ)] after drilling thin plates of Ti6Al4V and AA 2024-T3 alloys under different cutting speeds and feed rates are shown in Tables 1 and 2 , respectively.

From Table 1 can be observed that in general the ACC and FZ signals increased with the cutting speed when machining the Ti6Al4V alloy plate, irrespective of the feed rate employed, whereas the POW values decreased with increase in cutting speed. AE and POW parameters were more sensitive to variation in feed rate than others. AE signals tended to stabilize for speeds in excess of $15.5 \mathrm{~m} / \mathrm{min}$, regardless the feed rate employed. With regard the diameter, in general, it experienced different pattern for different combination of cutting speed and feed rate under the conditions investigated. The highest values of FZ values 
were obtained after machining with the lowest feed rate of $0.25 \mathrm{~mm} / \mathrm{rev}$, contrary to expected and usually reported in the literature.

In Table 2, results for AA 2024-T3 alloy show that the AE, ACC, POW and FZ signals increased with cutting speed. With regard the hole diameter, no significant influence of cutting speed and feed rate was observed on the diameter. From literature it is known that an increase in feed rate directly increases the primary and secondary shear zones, tool-chip contact length and, consequently, lead to increase in heat generation [29]. As a result of that, the yield strength of the tool material drops rapidly, thus accelerating wear at the cutting edge during machining. Machining the aluminum alloy with the highest feed rate was also responsible for the highest values of all the parameters investigated, with exception of FZ. Contrary to expected, the lowest values of the $\mathrm{Z}$ component force (FZ) were obtained after machining both AA 2024-T3 and Ti6Al4V alloys with the highest feed rate value of $0.125 \mathrm{~mm} / \mathrm{rev}$. Also, from Tables 1 and 2 can be observed that values obtained for most of parameters when machining the AA 2024-T3 were slightly higher than those for Ti6Al4V alloy. Since the experimental trials were carried out under dry condition, the high values of the hole diameters in particular for the AA 2024-T3 alloy may be attributed to the severe plastic deformation during it machining, thus generating great amount of heat at the cutting interface and, consequently, leading to the strength reduction and possible melt of the material in contact with the drill. The results are larger diameter and distortion. With regard the results for titanium alloy, although machining of titanium and its alloys has been a challenger, especially due to their high chemical affinity with most of commercially available cutting tools and their poor thermal conductivity, in this work low cutting speeds were employed with carbide drill, compared to the usually values reported in the literature. Under these conditions the tool wear rate was still low for the number of machined holes, reason for the lower variation in hole diameters after machining this alloy in comparison with that for aluminum alloy under the conditions investigated.

The next subsections present the network architectures and parameters selected for the three types of networks under study after running the algorithms described in Sect. 3, pointing out the ones that achieved the lowest mean absolute error of inner and outer diameters for each network after training. This is followed by a presentation of the results based on the criteria described in Sect. 3.3 for the evaluation of groups of input signals that were the most efficient in each of the evaluated networks.

\subsection{Results for the MLP network}

\subsubsection{MLP network with all the input signals}

The architecture of the MLP network with the best result for Ti6Al4V and AA 2024-T3 alloys is shown Fig. 8, which has six sensor signals, two cutting parameters and the drill diameter as inputs, three hidden layers, and two outputs corresponding to the minimum and maximum diameters. For the Ti6Al4V alloy, after running the algorithm shown in Fig. 4, the lowest mean maximum and minimum errors were obtained by a network with 10 neurons in the three hidden layers, with a 0.3 momentum and 0.7 learning rate, which was indicated as $\left[\begin{array}{lll}10 & 10 & 10\end{array}\right]$ Ti-6Al-4V alloy. For the AA 2024-T3 alloy, the lowest mean errors were obtained by a network with 20,10 and 5 neurons in each hidden layer, respectively. This configuration showed a momentum
Fig. 8 Representation of the architecture of the MLP network used for the two alloys of the test specimens with all the signals of the available sensors, the cutting parameters and the drill diameter

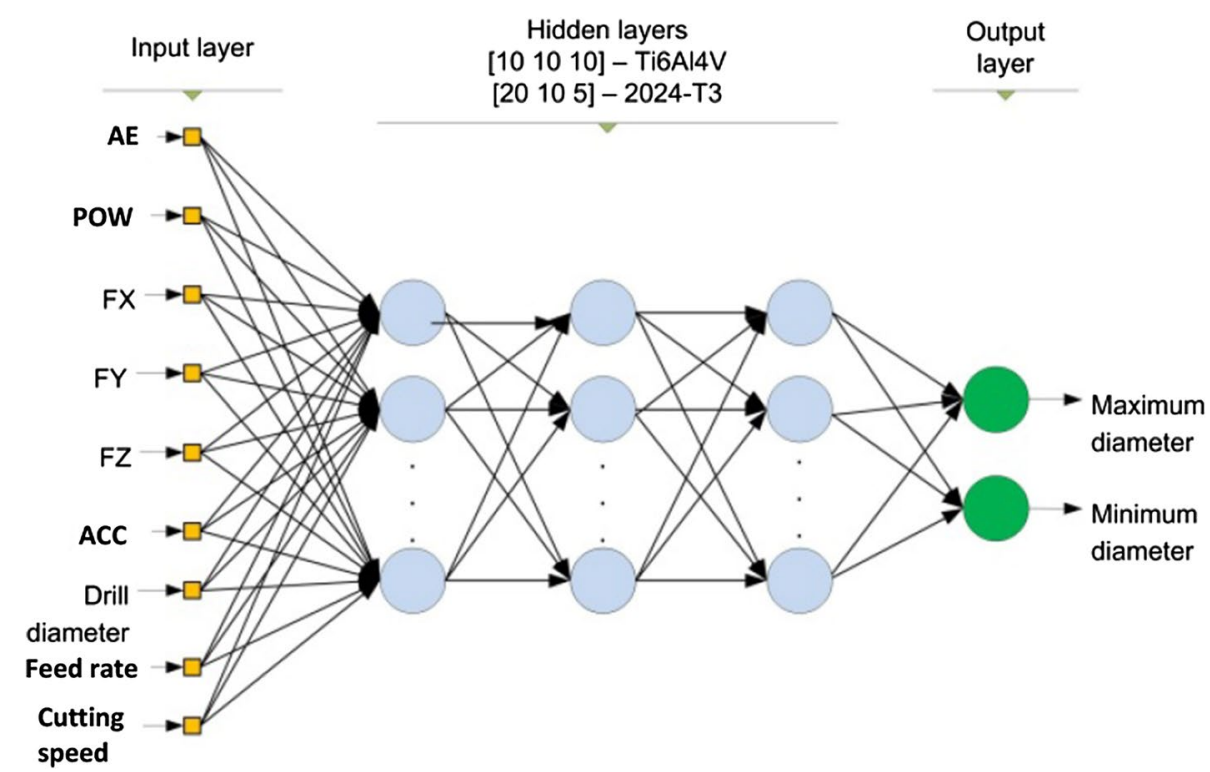



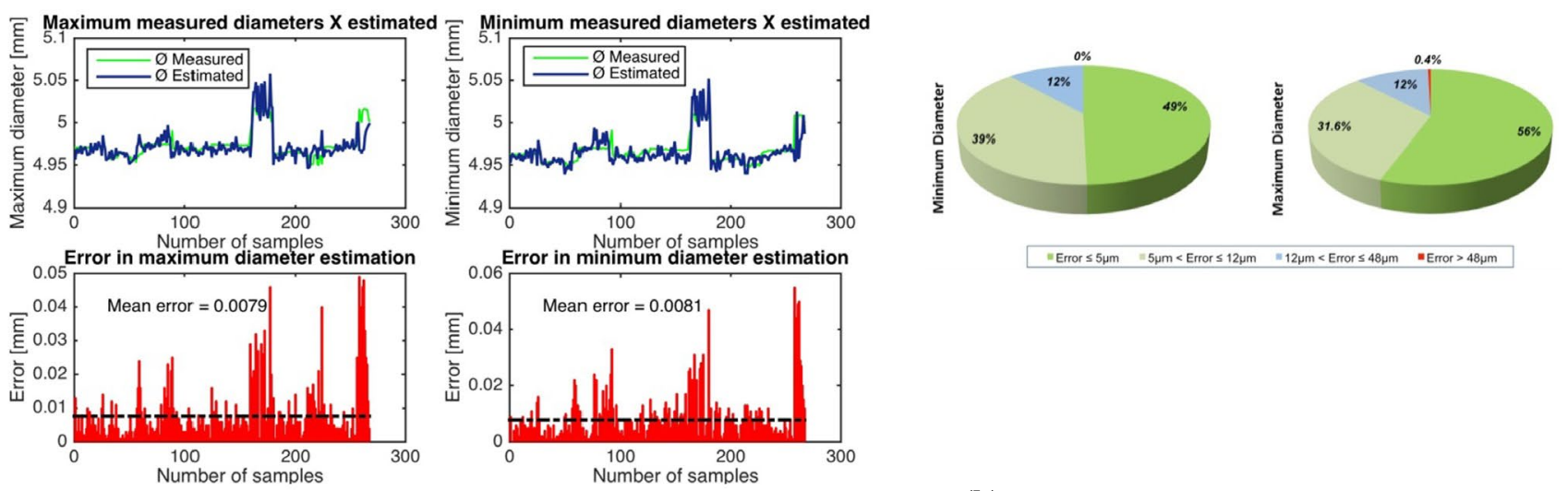

(a) ER for the MLP network using all the input signals for the Ti6Al4V

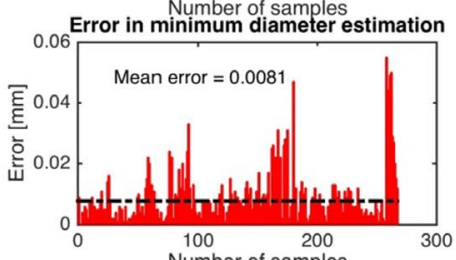

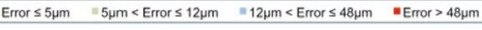

(b) EC in the MLP network using all the input signals for the Ti6Al4V alloy
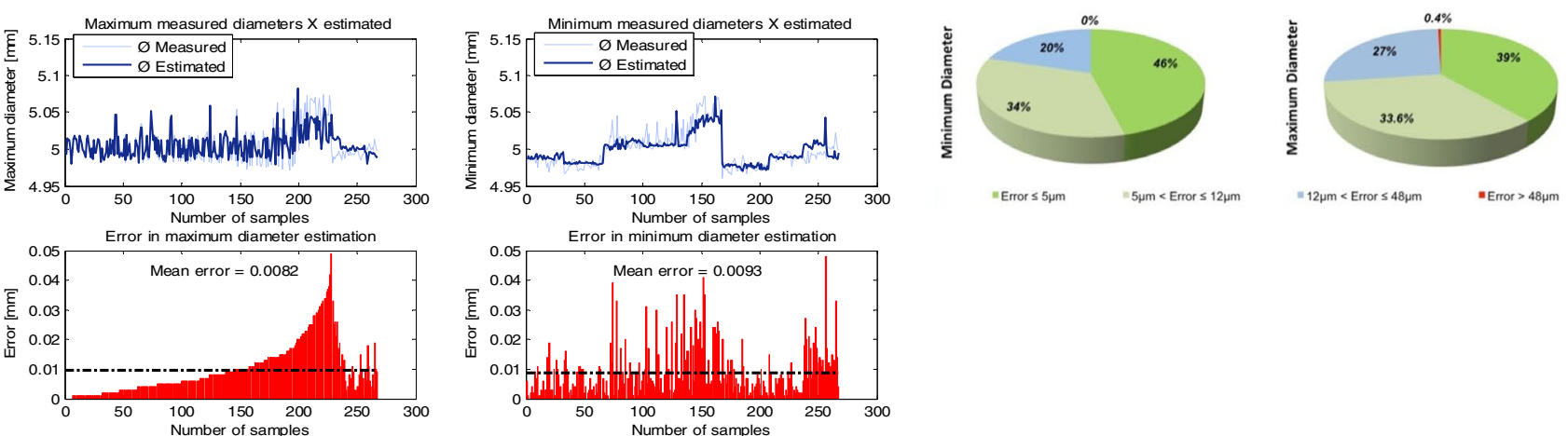

(c) ER for the MLP network using all the input signals for the AA 2024T3 alloy

(d) EC in the MLP network using all the input signals for the AA 2024-T3 alloy

Fig. 9 Estimation Results (ER) and Errors Classification (EC) in the estimation of hole diameters provided by the MLP network with all the input signals for the Ti6Al4V and AA 2024-T3 alloys

of 0.8 and a learning rate of 0.3 . Figure 8 illustrates the [20 10 5] architecture for AA 2024-T3.

Figure 9a shows the estimation results (ER) of the MLP network for Ti6Al4V alloy. It can be noticed that the values of 0.0079 and 0.0081 correspond to the mean estimation errors for the maximum and minimum diameters, respectively.

The analysis based on the quality control criterion indicated the ability of the MLP network to perform dimensional analysis adequately based on the measured diameters when using all the input signals, indicating the acceptance or rejection of a machined hole (Table 3 ).

The results obtained with the third criterion were assessed for correct estimates, according to criterion number two, i.e., ignoring the results that presented false positives or negatives.

Figure $9 \mathrm{~b}$ shows that $49.0 \%$ of the errors made by the MLP network in estimating the minimum diameter are undetectable, and that the percentage obtained for the maximum diameter was $56.0 \%$. This network performed satisfactorily in providing dimensional results, as indicated by the absence of errors in minimum diameter exceeding $48 \mu \mathrm{m}$ and by the presence of only $0.4 \%$ of error above the basic mechanical limit, which corresponds to the result of only one measurement for a set of 249 measurements of the hole diameter.

Figure $9 \mathrm{c}$ shows the results of the estimation by the MLP network for the AA 2024-T3 alloy. As can be seen, the values of the mean estimation error of the maximum and minimum diameters were 0.0082 and 0.0093 , respectively.

Based on the quality control criterion, the MLP network indicated that it was capable of performing the dimensional analysis satisfactorily based on the measured diameters, indicating the acceptance or rejection of a machined hole (Table 4). As can be seen in Table 4, after calculating the arithmetic mean between values of minimum $\left(D_{\min }\right)$ and maximum $\left(D_{\max }\right)$ diameters, i.e. on average, the results indicate that the network estimated $12.9 \%$ of the holes incorrectly, with $9.0 \%$ false positives and $3.9 \%$ false negatives when using all the input signals. 
Table 3 Behavior of the different networks using different input signals in the quality control evaluation of holes in the Ti6Al4V alloy

\begin{tabular}{|c|c|c|c|c|c|}
\hline \multirow[t]{2}{*}{ Network } & \multirow[t]{2}{*}{ Input signal } & \multicolumn{2}{|l|}{$D_{\mathrm{MIN}}$} & \multicolumn{2}{|l|}{$D_{\text {MAX }}$} \\
\hline & & & $\%$ & & $\%$ \\
\hline \multirow[t]{9}{*}{ MLP } & \multirow[t]{3}{*}{ All } & False positive & 1.1 & False positive & 1.5 \\
\hline & & False negative & 6.0 & False negative & 5.6 \\
\hline & & False results & 7.1 & False results & 7.1 \\
\hline & \multirow[t]{3}{*}{$\mathrm{FZ}$} & False positive & 0.7 & False positive & 0.7 \\
\hline & & False negative & 3.0 & False negative & 3.7 \\
\hline & & False results & 3.7 & False results & 4.5 \\
\hline & \multirow[t]{3}{*}{$\mathrm{AE}+\mathrm{ACC}$} & False positive & 6.0 & False positive & 4.1 \\
\hline & & False negative & 1.9 & False negative & 3.7 \\
\hline & & False results & 7.9 & False results & 7.9 \\
\hline \multirow[t]{3}{*}{ ANFIS } & \multirow[t]{3}{*}{$\mathrm{FZ}$} & False positive & 1.9 & False positive & 0.7 \\
\hline & & False negative & 2.2 & False negative & 4.9 \\
\hline & & False results & 4.1 & False results & 5.6 \\
\hline \multirow[t]{3}{*}{$\mathrm{RBF}$} & \multirow[t]{3}{*}{ All } & False positive & 0.4 & False positive & 2.2 \\
\hline & & False negative & 7.5 & False negative & 5.2 \\
\hline & & False results & 7.9 & False results & 7.5 \\
\hline
\end{tabular}

Table 4 Behavior of the different networks using different input signals in the quality control evaluation of holes in the AA 2024-T3 alloy

\begin{tabular}{|c|c|c|c|c|c|}
\hline \multirow[t]{2}{*}{ Network } & \multirow[t]{2}{*}{ Input signal } & \multicolumn{2}{|l|}{$D_{\mathrm{MIN}}$} & \multicolumn{2}{|l|}{$D_{\text {MAX }}$} \\
\hline & & & $\%$ & & $\%$ \\
\hline \multirow[t]{9}{*}{ MLP } & \multirow[t]{3}{*}{ All } & False positive & 7.5 & False positive & 10.5 \\
\hline & & False negative & 3.7 & False negative & 4.1 \\
\hline & & False results & 11.2 & False results & 14.6 \\
\hline & \multirow[t]{3}{*}{$\mathrm{FZ}$} & False positive & 3.4 & False positive & 9.4 \\
\hline & & False negative & 21.0 & False negative & 16.1 \\
\hline & & False results & 24.3 & False results & 25.5 \\
\hline & \multirow[t]{3}{*}{$\mathrm{POW}+\mathrm{FZ}$} & False positive & 4.1 & False positive & 7.5 \\
\hline & & False negative & 7.5 & False negative & 7.5 \\
\hline & & False results & 11.6 & False results & 13.3 \\
\hline \multirow[t]{3}{*}{ ANFIS } & \multirow[t]{3}{*}{$\mathrm{FZ}$} & False positive & 3.0 & False positive & 8.6 \\
\hline & & False negative & 12.4 & False negative & 13.5 \\
\hline & & False results & 15.4 & False results & 22.1 \\
\hline \multirow[t]{3}{*}{$\mathrm{RBF}$} & \multirow[t]{3}{*}{$\mathrm{FZ}$} & False positive & 7.9 & False positive & 7.5 \\
\hline & & False negative & 8.2 & False negative & 14.2 \\
\hline & & False results & 16.1 & False results & 21.7 \\
\hline
\end{tabular}

Figure $9 \mathrm{~d}$ indicates that $46 \%$ of the errors made by the MLP network in estimating the minimum diameter are undetectable, and that the percentage obtained for the maximum diameter was $39 \%$. The performance of this artificial intelligence system proved to be excellent since it indicated the dimensional results satisfactorily, as can be observed from the absence of errors exceeding $48 \mu \mathrm{m}$ in the minimum diameter and in the presence of an error of only $0.4 \%$ above the basic mechanical limit. Again, this corresponds to the result of only one measurement for a set of 238 hole diameter measurements. Thus, like the network for the Ti6Al4V alloy, the MLP network for the AA 2024-T3 alloy showed a single measurement with a deviation exceeding $48 \mu \mathrm{m}$.

Ferreiro et al. [25] employed data mining techniques to improve the detection of burr on holes drilled on an aerospace aluminum alloy, Al 7075-T6 grade. The experiments were carried out with three-edged carbide drills of $10 \mathrm{~mm}$ diameter, feed rate in rage of $0.2-0.5 \mathrm{~mm} / \mathrm{rev}$ and without coolant. The output parameters were the torque of electrospindle, electrical power consumed and axial force, especially because the signal of torque in particular has demonstrated good correlation with burr size. The objective was to obtain a more suitable classification model, with a result of Burr $=$ yes|Burr $=$ no, or in classes as Class $($ Burr $=$ no $)$ : admissible burr and Class $($ Burr $=$ yes $)$ : non admissible burr, as defined by aeronautical industry, based on the output parameters investigated and using different algorithms. After applying a tenfold cross-validation that was repeated ten times to calculate the accuracy of the prediction models., the authors observed that false negatives varied between 0 and 3 for all the machine learning algorithms, with type classification that includes classification trees, induction rules, distance based techniques, techniques based on probabilities and neural networks. They also found that J48 (classification trees type) and JRip (induction rules type) algorithms provided superior performance and were able to eliminate false negatives.

Bustillo et al. [30] have investigated the use of ensemble algorithms to achieve an accurate prediction model (based on a $10 \times 10$-fold cross-validation method) for surface roughness of a quenched steel (AISI H13) grade after ballend milling operation under various operational conditions (cutting speed, feed rate, radial depth of cut, tool properties geometry and machining environment). They reported that the best results were obtained after using a neural network with default training values ( 0.3 learning rate, 0.2 momentum, number of epochs $=500$ that resulted in an accuracy of $81.88 \%$ ) in comparison with the extensively fine-tuned neural network. Random subspace ensemble yielded better results than the fine-tuned MLP. The worse results were obtained after using the AdaBoost algorithm with experimental dataset. Finally, they observed that the best result of accuracy rate provided for a network was $83.03 \%$, obtained with parameters momentum of 0.075 , learning rate of 0.15 and number of epochs of 500 .

Cruz et al. [5] carried out an experimental and modelling investigation to estimate hole diameter and finishing in sandwich plates composed of Ti6Al4V and AA 2024T3 alloys after drilling operation under similar cutting conditions as those employed in the present study. Hole 
diameter and surface roughness values of each hole were the output variables for the experimental trials and also to feed into the ANN output layer employed. According to the authors, this can enable the biases to be updated on a backpropagation feed-forward algorithm. The authors adopted the following strategy of percentage of obtained total data to obtain the ANNs estimation error: $60 \%$ used ANN training, $20 \%$ for validation and $20 \%$ for ANN test to confirm it satisfactory performance. As a result, the authors found that an average error for estimated $\mathrm{min} /$ max diameter was 6.9 and $9.3 \mu \mathrm{m}$ for the Ti6Al4V alloy and AA 2024-T3 alloy, respectively. In terms of surface roughness, the absolute average estimation error was $0.264 \mu \mathrm{m}$ which was considered very low for a control method.

\subsubsection{Performance of the MLP networks considering the influence of the input signals}

The network configurations selected for each alloy were tested to verify the influence of the signals and combinations of signals. The result for the Ti6Al4V alloy (Fig. 10) indicated that the use of the acoustic emission and acceleration signals as inputs of the MLP network (AE + ACC) reached the lowest mean error in the estimation of diameters. However, these signals led to a poorer performance when applied separately. Considered separately as an input to the network, the signal with information about the force on the $Z$ axis (FZ) led to the lowest mean error.

In the MLP network used for estimating the hole diameters in the AA 2024-T3 alloy, the use of electric power

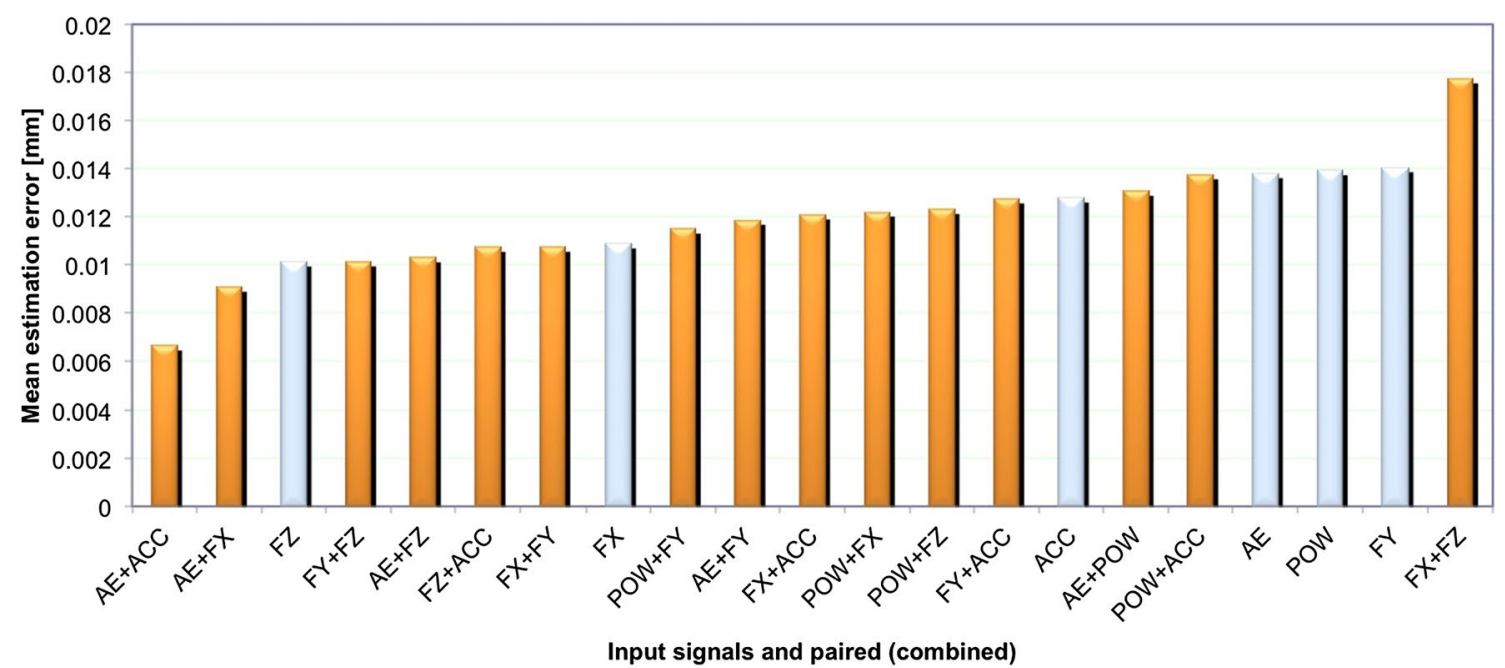

Fig. 10 Results of the test to verify the influence of separate signals and paired signals for the Ti6Al4V alloy

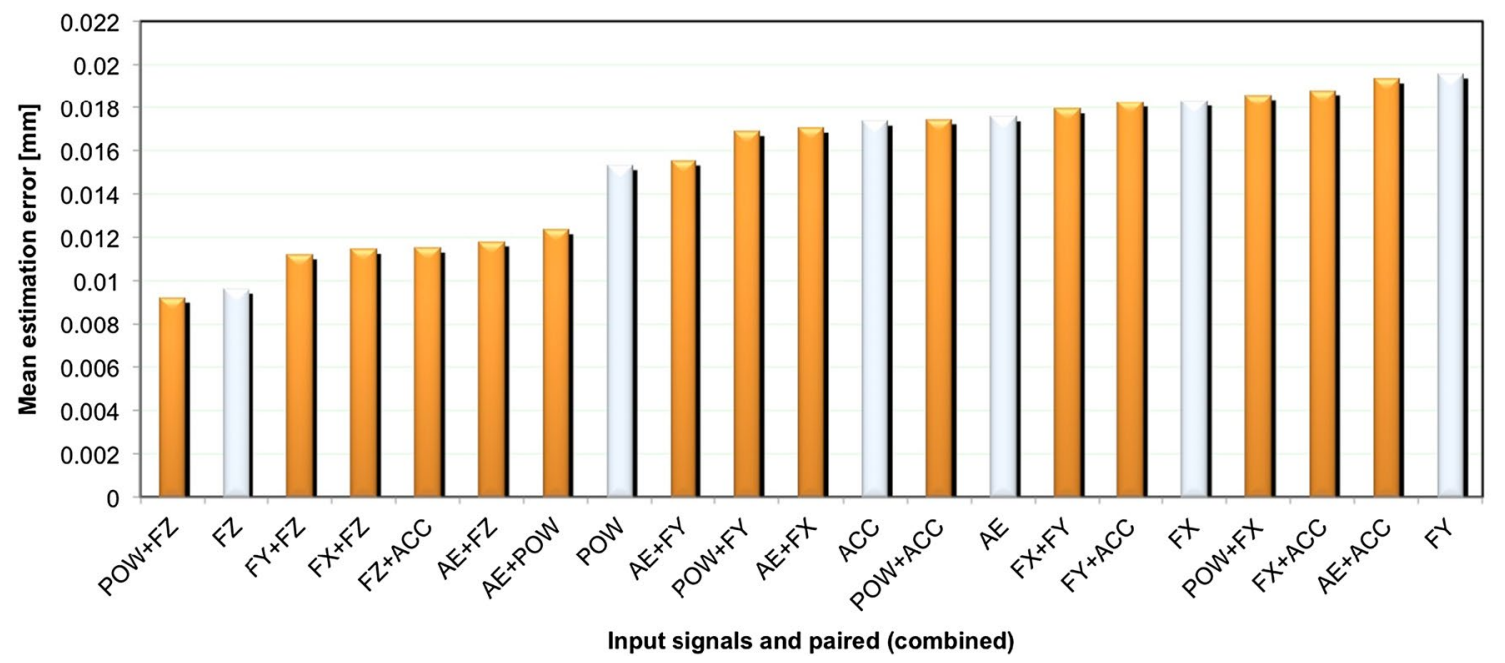

Fig. 11 Results of the test to verify the influence of separate signals and paired (combined) signals for the AA 2024-T3 alloy 
signals and $\mathrm{Z}$ component force (POW $+\mathrm{FZ}$ ) resulted in the lowest error among the set of inputs with two combined signals. Similarly to the result of the network for the Ti6Al4V alloy, the $\mathrm{Z}$ component force as a single input (FZ) produced the lowest error compared with the other signals applied separately as inputs (Fig. 11). It can be observed from Figs. 10 and 11 that in average the estimation error of diameter in the AA 2024-T3 alloy were slightly higher than that for AA 2024-T3 alloy. This can be attributed to higher values for most of output variables obtained after machining the latter that were used in training of the MLP network, thereby reflected on the predicted values.

The results from both the tests to verify the influence of the signals on the two materials indicate that two signals$\mathrm{Z}$ component force (FZ) and acoustic emission (AE) stand out in characterizing the drilling process when applied in combination with another signal. However, the comparison of the individual signals showed only the signal of $\mathrm{Z}$ component force standing out in the two networks. Therefore, the following tests were performed for the best configuration for an individual signal and for the configurations with the best combinations of signals.

\subsubsection{MLP network with the force $Z$ signal isolated}

The architecture employed for the analysis of the MLP network with the $\mathrm{Z}$ component force isolated as an input signal for both alloys is showed in Fig. 12. Figure 13a shows the results using only the signal of $\mathrm{Z}$ component force on Ti6Al4V alloy. It can be observed that the average estimation error was $0.0076 \mathrm{~mm}$ for the maximum diameter and $0.0078 \mathrm{~mm}$ for the minimum diameter. The percent relative error was low, corresponding to an average of only $0.2 \%$ for both diameters. The average errors highlighted in Fig. 13a were calculated from the point by point estimates of this configuration of the MLP. As can be seen, the darker line, which indicates the estimates of the MLP, followed the lighter line very accurately. The analysis based on the quality control criterion presented the results described in Table 3. These results indicate that, on average, the network estimated $4.1 \%$ of the holes incorrectly, with $0.7 \%$ false positives and $3.4 \%$ false negatives.

The result depicted in Fig. 13b indicates that $48 \%$ of the errors made by the MLP network in estimating the minimum diameter are undetectable, and that a percentage of $54 \%$ was obtained for the maximum diameter. The performance of the artificial intelligence system proved excellent in satisfactorily indicating the dimensional results. This is confirmed by the absence of errors exceeding $48 \mu \mathrm{m}$ for both the maximum and minimum diameters above the basic mechanical limit, which again corresponds to the results of only one measurement for a set of 255 hole diameter measurements.

The results for the AA 2024-T3 alloy indicate a lower mean error than that obtained by combining all the six available signals. The mean estimation error was $0.0095 \mathrm{~mm}$ for the maximum diameter and $0.0085 \mathrm{~mm}$ for the minimum diameter (Fig. 13c). The percent relative error was low, corresponding on average to only $0.2 \%$ for both diameters, like in the network for the Ti6Al4V alloy. The analysis based on the quality control criterion yielded the results presented in Table 4. These results indicate that, on average, the network incorrectly estimated $24.9 \%$ of the holes, with $6.4 \%$ false positive and $18.5 \%$ false negative.

The results shown in Fig. 13d indicates that $43 \%$ of the errors made by the MLP network in estimating the minimum diameter are undetectable, and that the percent obtained for the maximum diameter was $44 \%$. The performance of this artificial intelligence system proved to be excellent in satisfactorily indicating the dimensional results, as can be confirmed by the absence of errors exceeding $48 \mu \mathrm{m}$ for both the maximum and minimum diameters.

As reported in the literature, components of machining forces have presented a good correlation with geometrical errors [5, 7], burr size [25] and tool wear and tool failure [31-33], so the lowest error in estimation diameters verified when FZ was used input in MLP network in this study contributed to support such correlation.
Fig. 12 MLP network for the two alloys using the $\mathrm{Z}$ component force (FZ) as input signal

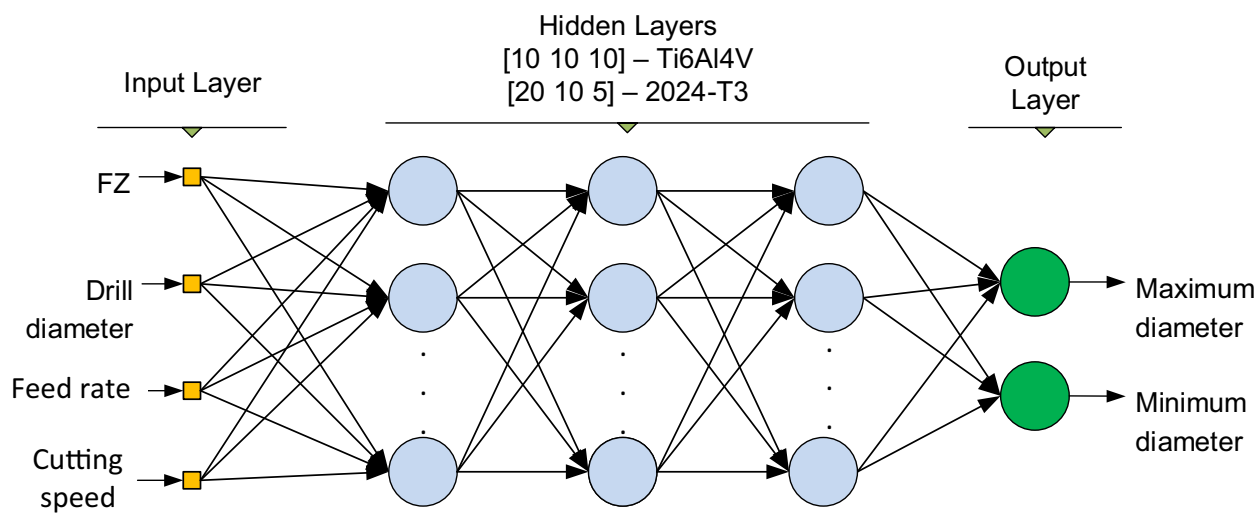



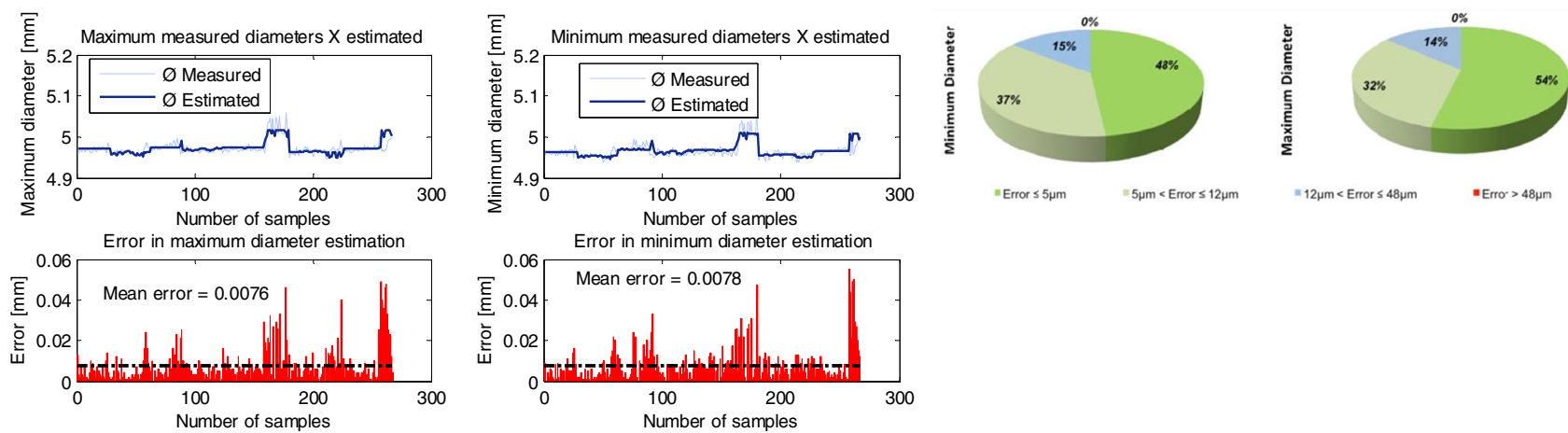

(a) ER for the MLP network using theFZ as input signal for the Ti6Al4V alloy
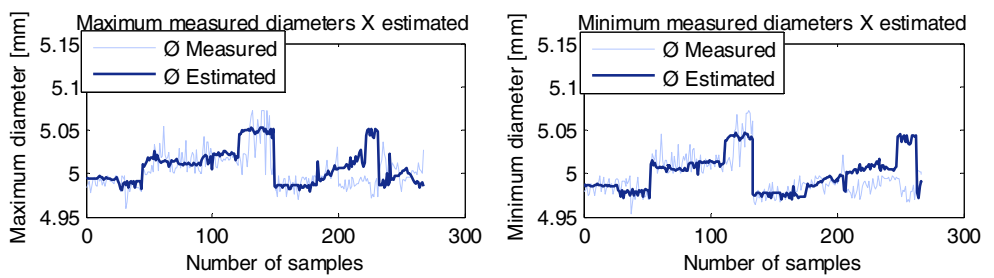

(b) EC in the MLP network using the FZ as input signal for the Ti6Al4V alloy
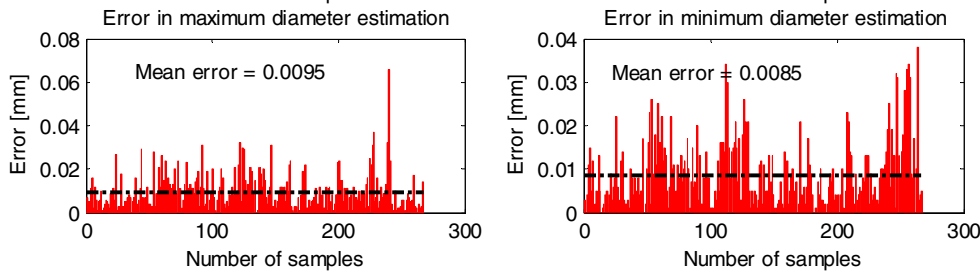

(c) ER for the MLP network using the FZ as input signal for the AA 2024-T3 alloy
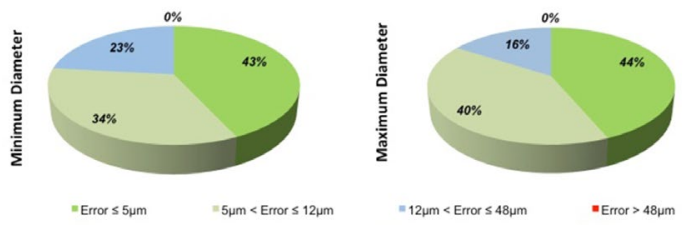

(d) EC in the MLP network using the FZ as input signal for the AA 2024-T3 alloy

Fig. 13 Estimation Results (ER) and Errors Classification (EC) in the estimation of hole diameters provided by the MLP network using the Z-component force (FZ) as input signal for the Ti6Al4V and AA 2024-T3 alloys

Fig. 14 Architectures used for testing the MLP networks using combination of two input signals

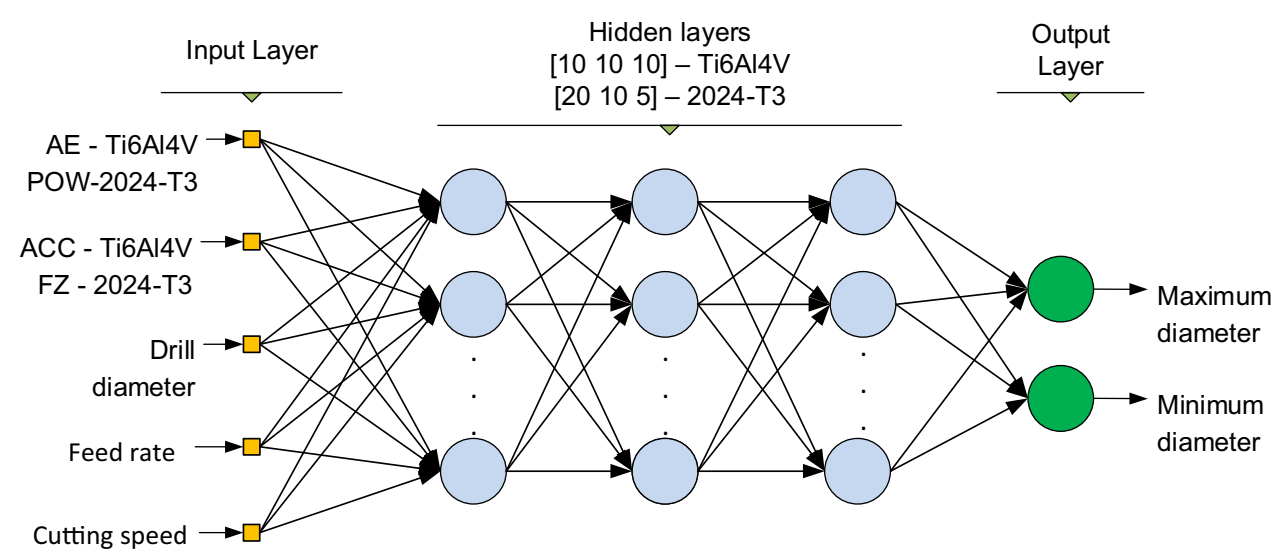

\subsubsection{MLP network with two input signals}

The results of the tests with different signals used as input signals for the MLP networks indicate that the acoustic emission (AE) signal combined with the acceleration signal (ACC) presented the lowest error in the estimates for the
Ti6Al4V alloy. The electric power (POW) and Z component force (FZ) signals, in turn, led to the best performance for AA 2024-T3 alloy, as illustrated in Figs. 10 and 11. The architectures used for a more detailed analysis are represented in Fig. 14. 

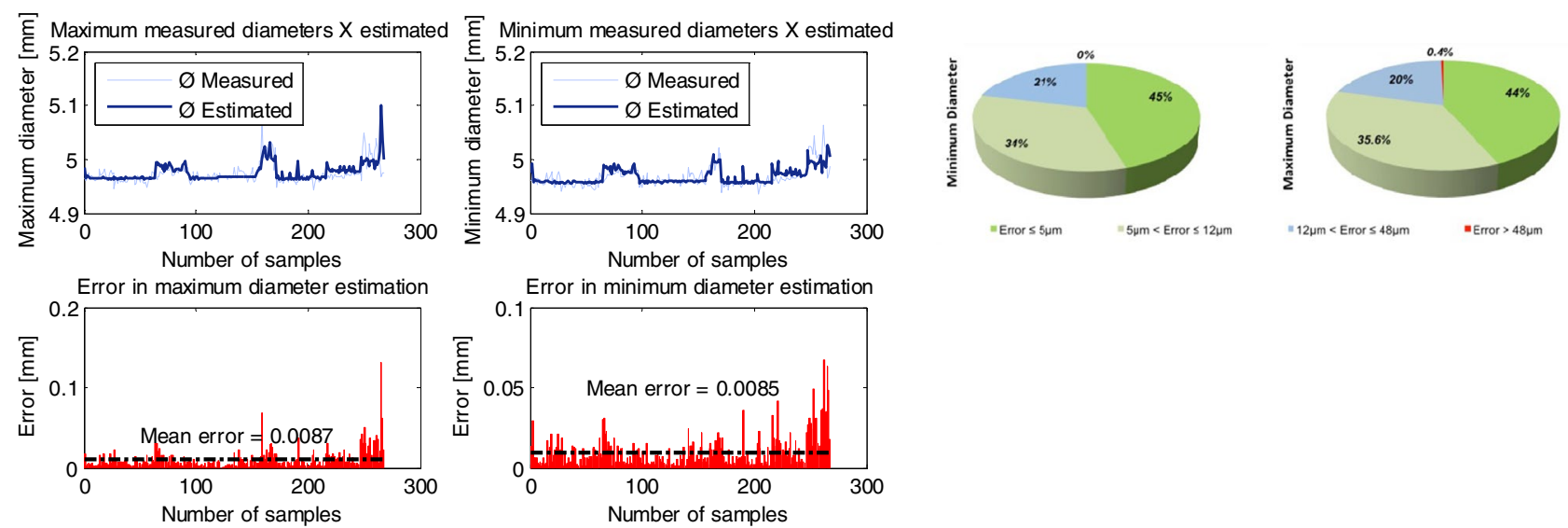

(a) ER for the MLP network using the AE and ACC as input signals for the Ti6Al4V alloy
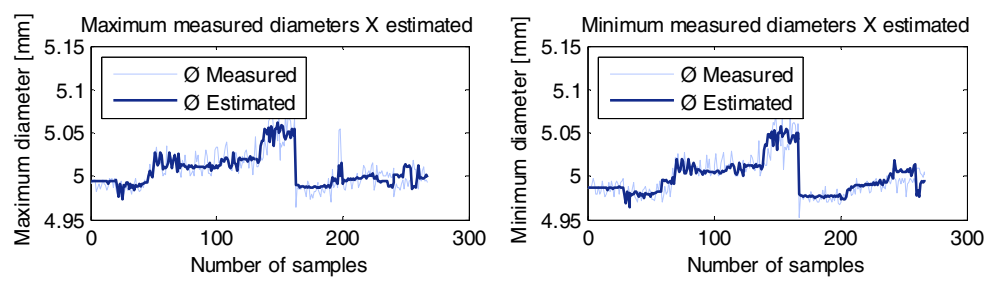

(b) EC in the MLP network using the AE and ACC as input signals for the Ti6Al4V alloy
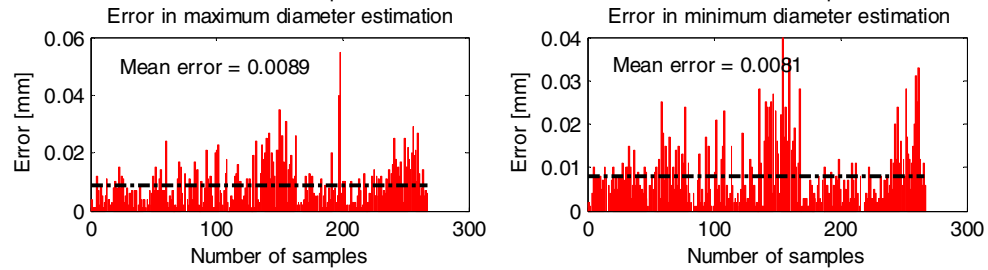

(c) ER for the MLP network using the POW and FZ as input signals for the AA 2024-T3 alloy
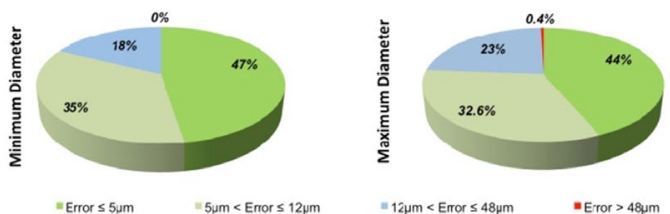

(d) EC in the MLP network using the POW and FZ as input signals for the AA 2024-T3 alloy

Fig. 15 Estimation Results (ER) and Errors Classification (EC) in the estimation of hole diameters provided by the MLP network with different combination of input signals for the Ti6Al4V and AA 2024-T3 alloys

The results in Fig. 15a indicate a mean error very similar to that achieved by the combination of all six available signals. The average estimation error was $0.0087 \mathrm{~mm}$ for the maximum diameter and $0.0085 \mathrm{~mm}$ for the minimum diameter. The percent relative error was low, corresponding on average to only $0.2 \%$ for both diameters. As can be seen, the darker line, which indicates the estimates of the MLP, accompanied the lighter line with a certain degree of accuracy. The analysis based on the quality control criterion yielded the results presented in Table 3 . These results indicate that, on average, the network estimated $7.9 \%$ of the holes incorrectly, with $5.1 \%$ false positive and $2.8 \%$ false negative when using the AE and ACC as input signals. These figures are slightly lower than those obtained showed in the configuration with all the signals used as input signals for the network.

With regard to the third criterion, the results depicted in Fig. $15 \mathrm{~b}$ indicate that $45 \%$ of the errors made by the MLP network in estimating the minimum diameter are undetectable, and that the error percentage for the maximum diameter was $44 \%$. The performance of this artificial intelligence system was excellent in satisfactorily indicating the dimensional results. This is confirmed by the absence of errors exceeding $48 \mu \mathrm{m}$ for the maximum diameter and only one measurement of the minimum diameter exceeding the basic mechanical limit in a set of 246 holes diameters measurements.

For the AA 2024-T3 alloy, using the paired signals of electric power (POW) and Z component force (FZ), the results (Fig. 15c) indicate a mean estimation error of $0.0089 \mathrm{~mm}$ for the maximum diameter and of $0.0081 \mathrm{~mm}$ for the minimum diameter. The percent relative error was low, corresponding on average to only $0.17 \%$ for both diameters. The analysis based on the quality control criterion yielded the results presented in Table 4. These results indicate that, on average, the network estimated $12.5 \%$ of the holes incorrectly, with $5.8 \%$ false positive and $7.5 \%$ false negative.

The analysis based on tolerance, with two input signals, indicates that $47 \%$ of the errors made by the MLP network 
in estimating the minimum diameter are undetectable, and that $44 \%$ correspond to the maximum diameter. The performance of this artificial intelligence system was considered excellent in indicating the dimensional results satisfactorily. This is confirmed by the absence of errors exceeding $48 \mu \mathrm{m}$ for the maximum diameter and only one measurement of the minimum diameter exceeding the basic mechanical limit in a set of 237 holes diameters measurements (Fig. 15d).

\subsection{Results obtained by the ANFIS System}

The results of the ANFIS system were obtained after running the algorithm described in Fig. 6. The influence of the signals on the system's performance was checked prior to tests.
In the case of the Ti6Al4V alloy, the FZ was clearly the signal with the most positive influence on performance, in terms of obtaining lower percentage errors. When combining two input signals, the best result was achieved by combining the data of the forces on the $X$ and $Z$ axes. The final classification is shown in Fig. 16.

Like the results obtained for the Ti6Al4V alloy, the results obtained for the test of the ANFIS system for the AA 2024-T3 alloy indicate that the force on the $Z$ axis is the signal that exerts the most positive influence. In the combination of two input signals, the best result was attained by combining the data of acoustic emission with the force on the $Z$ axis. Figure 17 shows the final classification. Again, a component of machining force (FZ) confirmed its good

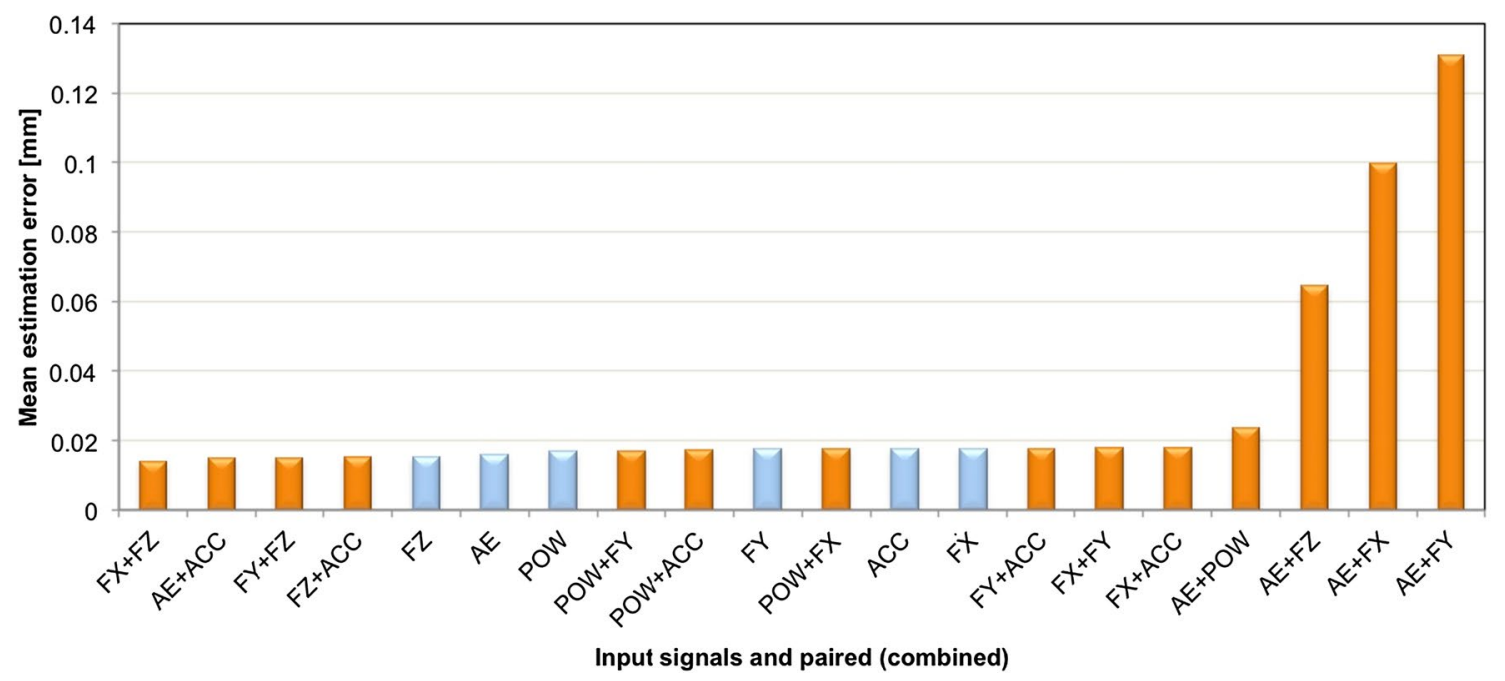

Fig. 16 Results of the test to verify the influence of individual and combined signals on the ANFIS system for the Ti6Al4V alloy

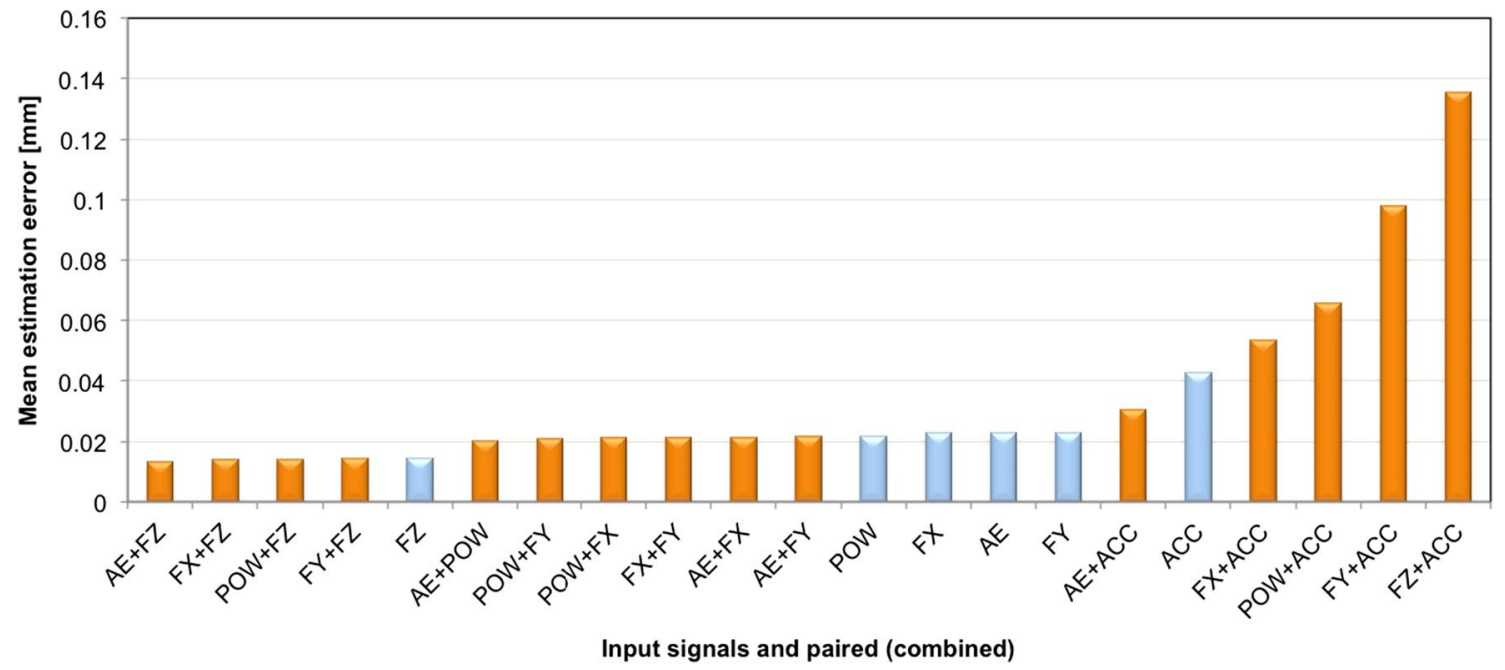

Fig. 17 Results of the test to verify the influence of the individual and combined signals on the ANFIS system for the AA 2024-T3 alloy 
correlation to estimate the hole diameters in both Ti6Al4V and AA 2024-T3 alloy with AFNIS system, like observed with MLP network.

The test to determine the number of epochs required for training was evaluated using the algorithm in Fig. 6. The result indicates that 105 epochs were required to train the system for the Ti6Al4V alloy and 270 epochs were needed to train it for the AA 2024-T3 alloy.

Figure 18 depicts the result of this test, clearly showing the difference in the behavior of the error for the two alloys investigated. It can be seen that the absolute error for Ti6Al4V alloy abruptly decreases from 0.11 to 0.038 when number of epochs is increased up to 40 , then it remains practically steady until to reach the number of epoch of 270 , point that it decreases again to the value of 0.017 and finally keeps stable until the end. Contrarily to the observed for the Ti6Al4V alloy, absolute error for the AA 2024-T3 alloy remained steady, regardless of the number of epochs used in training. This divergence may be attributed to the microstructural differences between the Ti6Al4V alloy and the AA 2024-T3 alloy, whose low complexity resulted in negligible variations in the collected signals, thus simplifying their mapping. The values obtained in this test were used in the subsequent tests. It is known from literature that titanium and its alloys are classified as difficult-tomachining materials with conventional tools, especially because the main elements present in titanium alloys, such as $\mathrm{Ti}$ and $\mathrm{Al}$, have high chemical affinity with most cutting tool materials such HSS, cemented carbide, ceramics and CBN/PCBN. Also, due to the high diffusion rate and instability of the segmented chip formation process when machining titanium alloys, tools are subjected to accelerated wear [34, 35], specifically when machining without coolant (what is not a recommended practice in industrial applications), like in this study. This adversely affects the dimensional and form errors. Therefore, the ANFIS system was able to detect the poor machinability of the Ti6Al4V

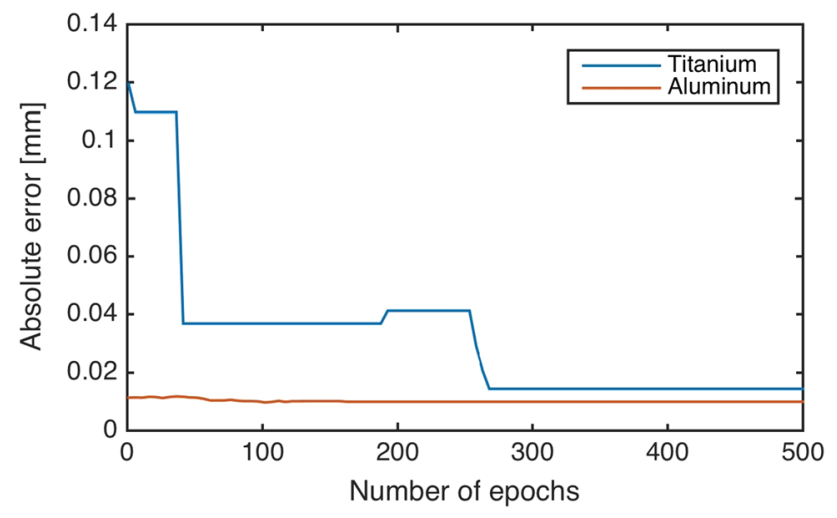

Fig. 18 Results of the test to ascertain the influence of the number of epochs on the ANFIS system for each alloy alloy in comparison with the AA 2024-T3 alloy under the conditions investigated.

The three criteria defined in Sect. 3.3 were used to organize the results from the ANFIS system, following the same procedure as the one employed to analyse the performance of the MLP network.

\subsubsection{ANFIS system with Z component force signal applied as input signal}

Table 5 lists the parameters of the clusters, which were defined by trial and error for both alloys until the lowest possible error was obtained. After configuring the system, the results of the estimates were obtained and are depicted in Fig. 19a. The mean absolute error in estimating the diameter was $0.0090 \mathrm{~mm}$, with a mean percentage error of $0.3 \%$. As for the quality control criterion, the ANFIS system showed good efficacy by presenting an average of only $4.9 \%$ of false results in the assessment of hole dimensions. Also on average, $1.3 \%$ corresponds to false positives and $3.6 \%$ to false negatives when using $\mathrm{Z}$ component force as input signal. The results for the minimum and maximum diameters in the Ti6Al4V alloy are shown in Table 3.

For the third criterion, the error classification indicates that, on average, $42.0 \%$ of the estimates had an error lower than the precision of the measuring instrument. None of the estimates of the minimum diameter exceeded the limit of precision mechanics, while only one estimate for the maximum diameter exceeded this limit, as shown in Fig. 19b.

For the AA 2024-T3 alloy, the signal cluster with the lowest mean error in estimating the diameter was also the isolated $\mathrm{Z}$ component force signal. Table 6 describes the cluster parameters for the ANFIS system used for estimating of the hole diameters in the AA 2024-T3 alloy.

The results from the estimates were obtained after configuring the system, and are illustrated in Fig. 19c. The mean absolute error in estimating the diameter was $0.0102 \mathrm{~mm}$, with a mean percentage error of $0.4 \%$. As for the quality control criterion, the ANFIS system showed reasonable efficiency by presenting, on average, only $18.9 \%$ of false results in the evaluation of hole dimensions. Also on average, $5.8 \%$ corresponds to false positives and $13.0 \%$ to false negatives when using $\mathrm{Z}$ component force as input signal. The results for the minimum and maximum

Table 5 Parameters of influence of the cluster centers defined by the ANFIS system of estimation for the Ti6Al4V alloy

\begin{tabular}{ll}
\hline Parameter & Value \\
\hline Radius of influence of the cluster center & Input cluster $=0.12$ \\
& Output cluster $=0.12$ \\
Distance between the cluster centers & 1.25 \\
\hline
\end{tabular}



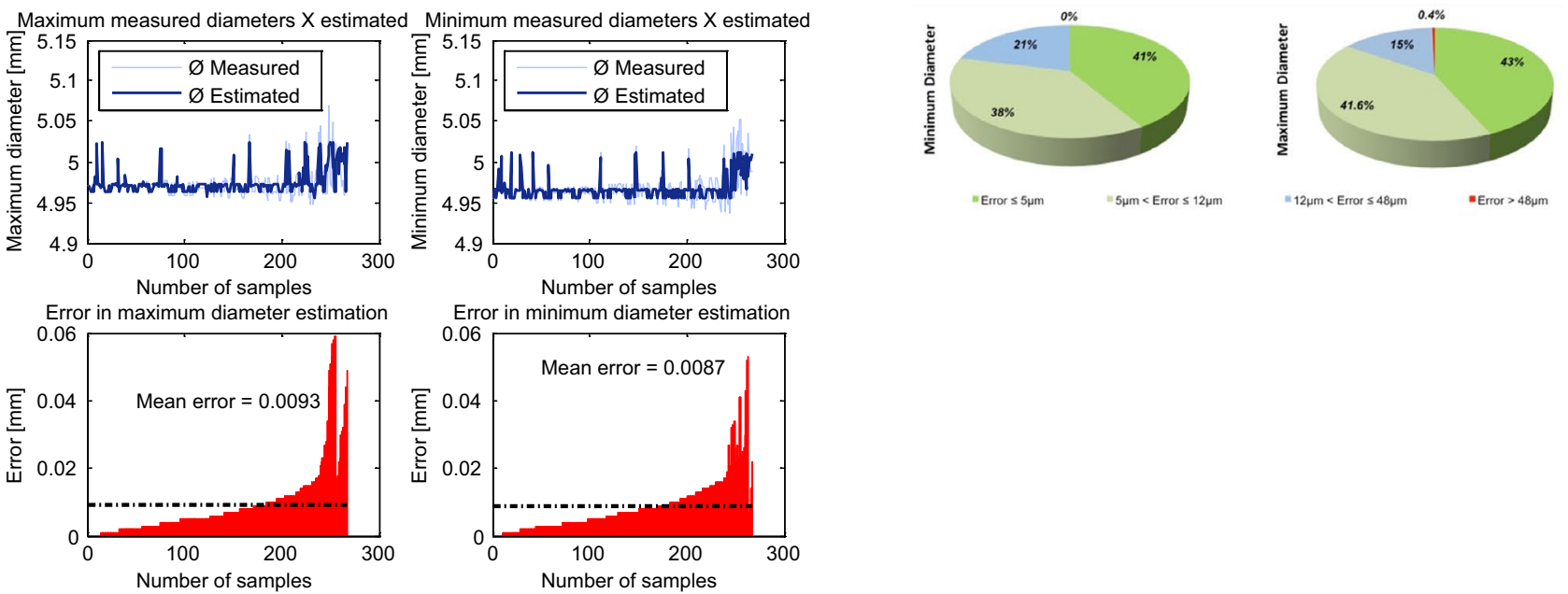

(a) ER for the ANFIS system using the FZ as input signal for the Ti6Al4V alloy
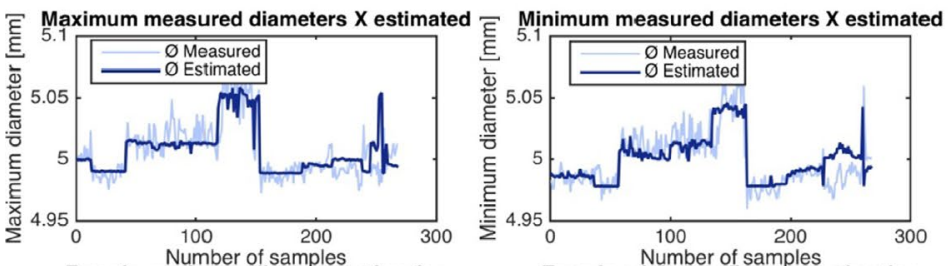

(b) EC in the ANFIS system using the FZ as input signal for the Ti6Al4V alloy
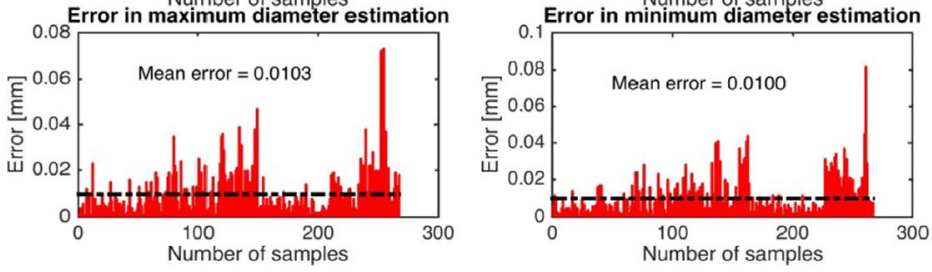

(c) ER for the ANFIS system using the FZ as input signal for the AA 2024-T3 alloy
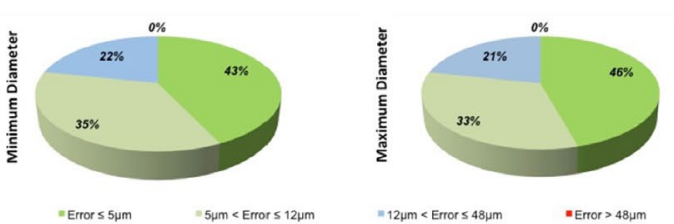

(d) EC in the ANFIS system using the FZ as input signal for the AA 2024-T3 alloy

Fig. 19 Estimation Results (ER) and Errors Classification (EC) in the estimation of hole diameters provided by the ANFIS system using the Z component force (FZ) as input signal for the Ti6Al4V and AA 2024-T3 alloys

Table 6 Parameters of influence of the cluster centers defined by the ANFIS system of estimation for the AA 2024-T3 alloy

\begin{tabular}{ll}
\hline Parameter & Value \\
\hline Radius of influence of the cluster center & Input cluster $=0.36$ \\
& Output cluster $=0.21$ \\
Distance between the cluster centers & 1.25 \\
\hline
\end{tabular}

diameters in AA 2024-T3 alloy when using $\mathrm{Z}$ component force as an input signal are shown in Table 4.

For the third criterion, the error classification indicates that, on average, $43.0 \%$ of the estimates had an error below the precision of the measuring instrument. None of the estimates exceeded the limit of precision mechanics, as can be seen in Fig. 19d.

\subsection{Results for the RBF network}

\subsubsection{RBF networks using all the input signals}

The group of signals that provided the lowest mean diameter estimation error was the set of all the collected input signals.

The RBF network was configured for the Ti6Al4V alloy using a spread of 80 , yielding the results presented in Fig. 20a. The absolute error for the two diameters was $0.0092 \mathrm{~mm}$, on average, while the percentage error was $0.3 \%$, as also shown in Fig. 20a. The analysis based on the quality control criterion indicates that, on average, the RBF network presented false results in $7.7 \%$ of the estimates, i.e., $1.3 \%$ false positives and $6.4 \%$ false negatives when using all the input signals (Table 3 ). 

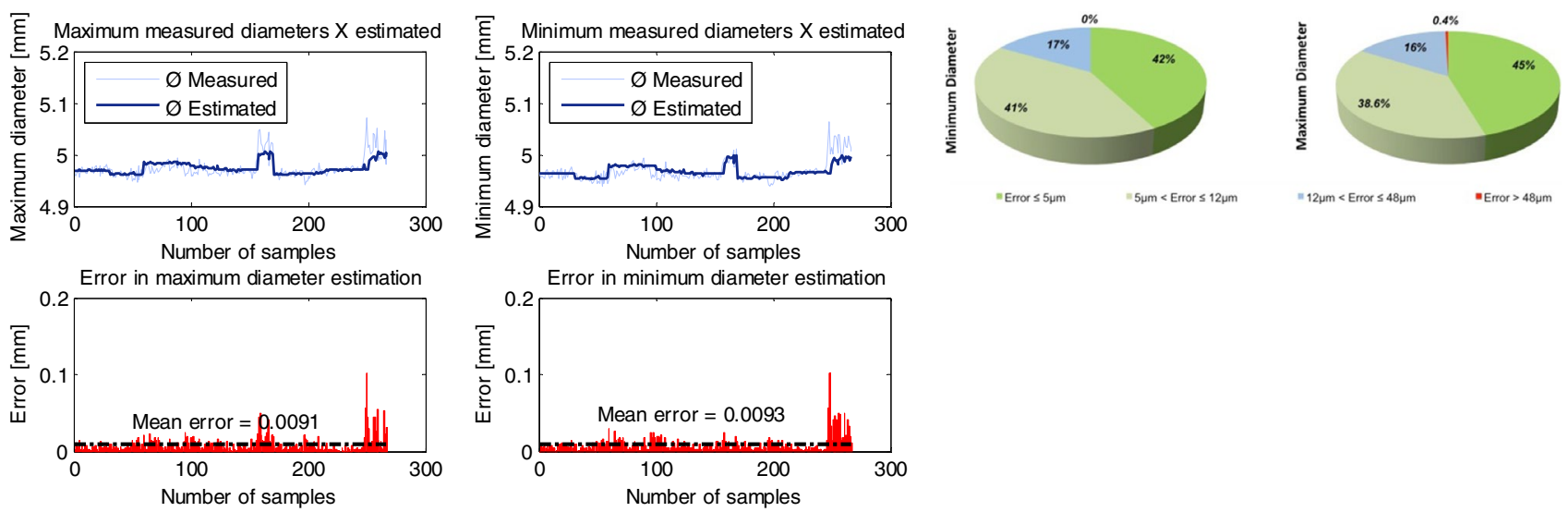

(a) ER for the RBF network using all the input signals for the Ti6Al4V alloy

(b) EC in the RBF network using all the input signals for the Ti6Al4V alloy
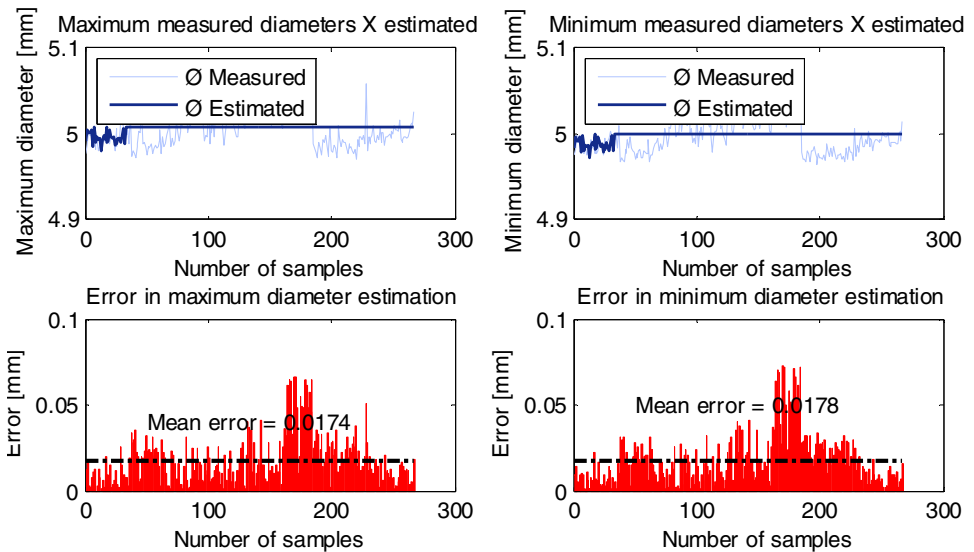

(c) ER for the RBF network using all the input signals for the AA 2024-

T3 alloy, which failed to present generalization

Fig. 20 Estimation results (ER) and errors classification (EC) in the estimation of hole diameters provided by the RBF network with all the input signals for the Ti6Al4V and AA 2024-T3 alloys

For the third criterion, the error classification indicates that, on average, the error of $43.5 \%$ of the estimates was below the precision of the measuring instrument. None of the measurements of the minimum diameter presented an error above the limit of precision mechanics (Fig. 20b), and only one measurement of the maximum diameter exceeded this limit, representing $0.4 \%$ of the estimates.

With a spread of 10,000 for the AA 2024-T3 alloy, the RBF network was configured for training and subjected to simulation. However, this configuration was not able to generalize from the training data, as can be seen in Fig. 20c. After initiating the variations in the measured diameters, the network continues to make estimates, but with a negligible variation, appearing to adopt a constant value. This behavior generated a relatively low mean error, which, in a first analysis, may lead to an erroneous pre-assessment about the performance of this network, which can be observed by analysing Fig. 20c. This detected problem invalidated the subsequent analyses, and it should be noted that $50.6 \%$ of the measuring results were incorrect.

It should be noted that the good performance of the RBF is tied to the amount of available training data, and that it requires a large amount of information due to the characteristics of its algorithm. Therefore, the results from this study indicate that a larger number of points are required than were used here, which is an obstacle intrinsic to the accurate determination of an ideal number, for this particular case.

\subsubsection{Evaluation of the influence of the signals and the spread}

The results from the influence tests presented in the flowchart in Fig. 7 show the positive influence of the signal of $\mathrm{Z}$ component force on the performance of the RBF network. When applied separately to the RBF network for the 


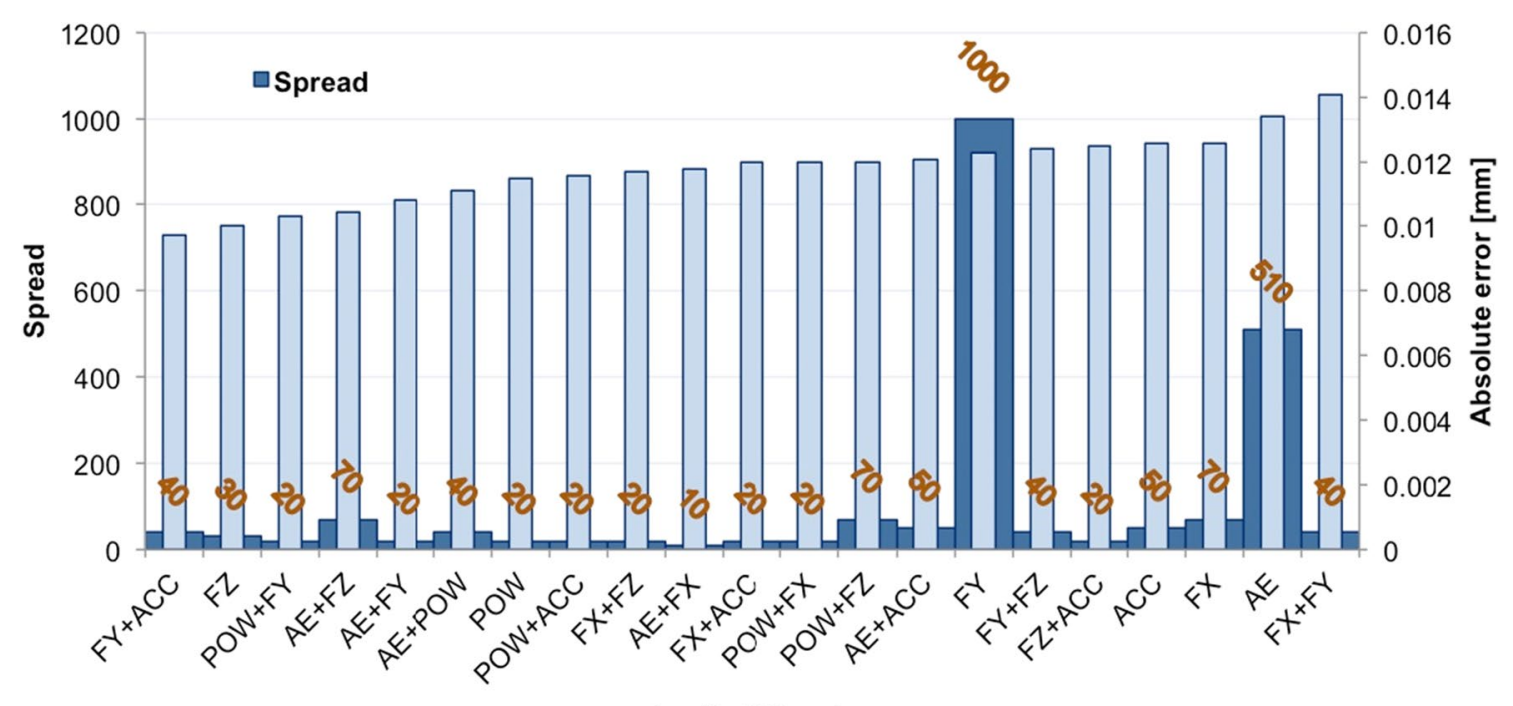

Applied Signals

Fig. 21 Results of the test to ascertain the influence of individual and paired signals, plus spread, on the performance of the RBF network for the Ti6Al4V alloy

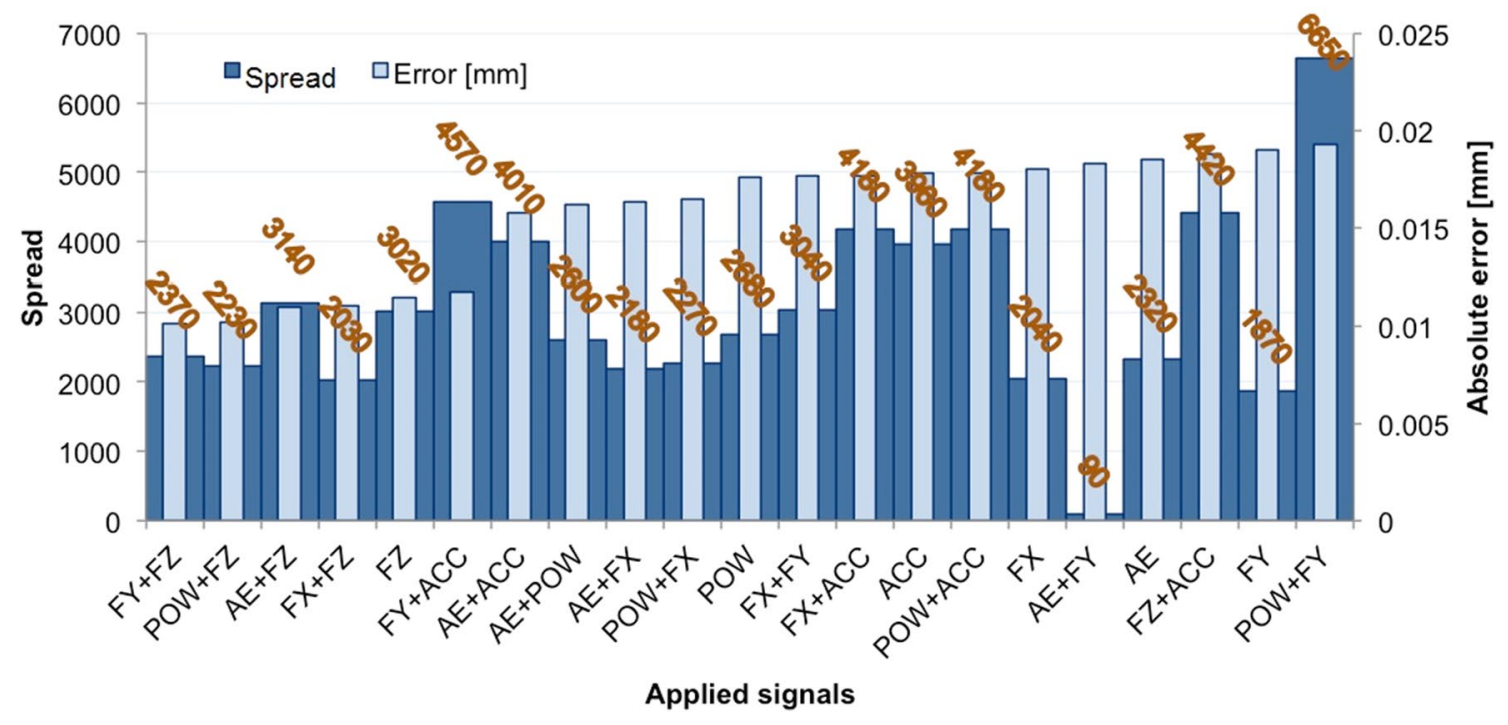

Fig. 22 Results of the test to ascertain the influence of individual and paired signals, plus spread, on the performance of the RBF network for the AA 2024-T3 alloy

Ti6Al4V alloy, the $\mathrm{Z}$ component force resulted in a mean error of $0.0100 \mathrm{~mm}$, using a spread of 30 . The paired signals with the lowest estimation error were the combination of the force on the $Y$ axis and the acceleration, which resulted in an average error of $0.0097 \mathrm{~mm}$, with a spread of 40. The complete classification is shown Fig. 21.

Applied separately in the RBF network for the AA 2024$\mathrm{T} 3$ alloy, the $\mathrm{Z}$ component force resulted in a mean error of $0.0115 \mathrm{~mm}$, using a spread of 3020 . The paired signals with the lowest estimation error were the combination of the force on the $Y$ axis and the force on the $Z$ axis, which resulted in a mean error of $0.0101 \mathrm{~mm}$, with a spread of 2370. The complete classification is shown in Fig. 22.

The size of state space hidden inside a RBF network is high and data classification problem tends to be linearly separable. This was one of the reasons for evaluating RBF networks. Whereas an artificial neural network can be developed as an approximation problem in a high-dimensional space, the learning setting in a RBF network aims to find a surface inside that space that best fits the training data. Generalization is equivalent to interpolation of the surface test data. The training algorithms performs the 

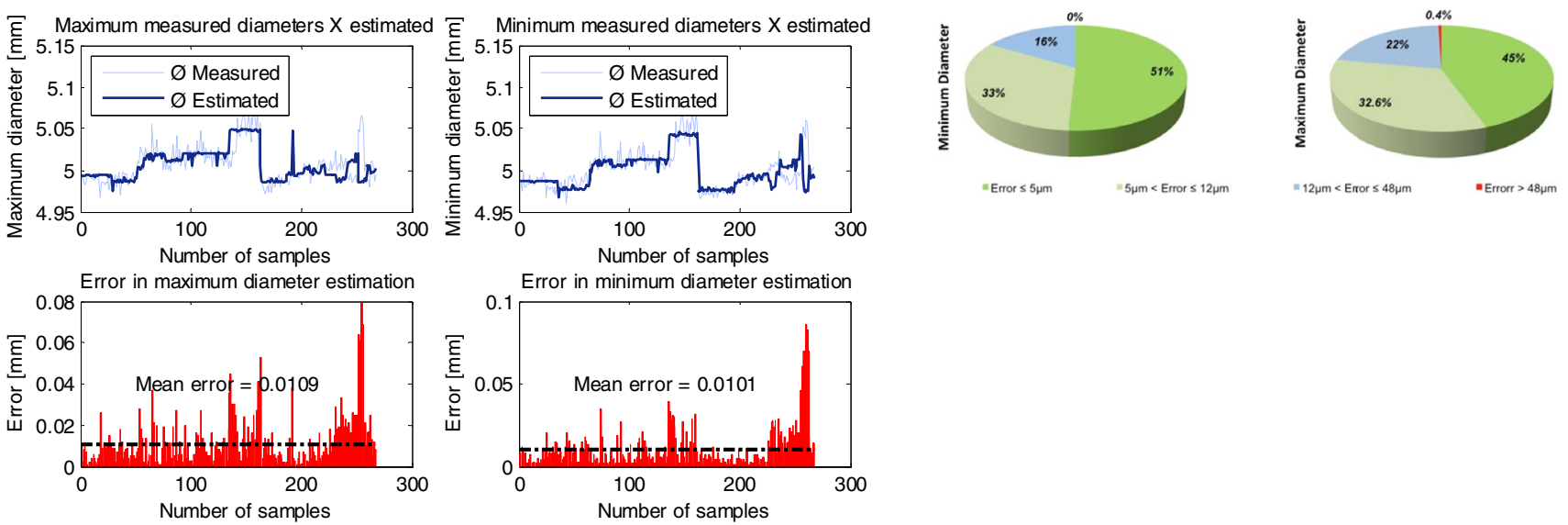

(a) ER for the RBF network using the FZ as input signal for the AA 2024-T3 alloy

(b) EC in the RBF network using the FZ as input signal for the AA 2024-T3 alloy

Fig. 23 Estimation Results (ER) and Errors Classification (EC) in the estimation of hole diameters provided by the RBF network using the Z-component force (FZ) as input signal for the Ti6Al4V and AA 2024-T3 alloy

adjustment of the centers as well as supports the radial basis functions in the hidden layer usually by a data clustering algorithm such as k-means, in a first step. The second step is to adjust the synaptic weights of the output layer, using backpropagation method. RBF network has faster training than MLP network, but it needs more data. The sensitivity of a RBF network may be tuned adjusting spread, where a large spread implies less sensitivity. As shown earlier, an algorithm was developed to test simultaneously the input signals and combinations of these signals in pairs together with spread. However, the spread test was also performed for all inputs together, for each material of the test body. The spread range for titanium alloy was the same as used in the algorithm (10-1000). However, for the aluminum alloy, this range did not show good results. Thus, the spread range used for the aluminum alloy was from 10 to 10,000 . It was observed that the error value increases when spread goes higher than 10,000 .

\subsubsection{RBF network using as input only the $Z$ component force signal}

For the AA 2024-T3 alloy, the RBF network that presented the lowest mean error in estimating the diameters with a single sensor signal was the individual $\mathrm{Z}$ component force. The mean error in estimating the maximum diameter was $0.0101 \mathrm{~mm}$ and that of the minimum diameter was $0.0109 \mathrm{~mm}$ (Fig. 23a). The percent relative error was low, corresponding on average to only $0.2 \%$ for both diameters. The analysis by the quality control criterion presented the results listed in Table 4. These results indicate that, on average, the network estimated $18.9 \%$ holes incorrectly, with $7.7 \%$ false positive and $11.2 \%$ false negative when using $\mathrm{Z}$ component force as input signal.
The mean errors listed in Table 4 for the $\mathrm{Z}$ component force signal were calculated from the point by point estimates of this configuration of the RBF network. In Fig. 23a, note that the darker line, which indicates the RBF estimates, followed the lighter line, but with a lower accuracy than the MLP network.

The results shown in Fig. 23b indicate that $51 \%$ of the errors made by RBF network in estimating the minimum diameter are undetectable, and that the percentage obtained for the maximum diameter was $45 \%$. Only one measurement of the maximum diameter exceeded the basic mechanical limit.

\subsection{Comparison of the performance of the three tested artificial intelligence systems}

All the results obtained in the tests and simulations of the three artificial intelligence systems involved in this study, as well as the results achieved with the groups of signals that did not obtain the lowest mean absolute error in the estimation of hole diameters for both the Ti6Al4V and AA2024-T3 alloys are shown in Table 7. The results for the two alloys were separated due to the divergent behavior of the signals elicited from each material.

In addition to the results described in detail in this paper, in Table 7 are also presented the results obtained with all the groups of simulated signals and which showed higher absolute errors than those already presented. The columns containing the sign "-" represent the system's failure to produce satisfactory results. It was found in the ANFIS system, that slight changes in the cluster of input and output data used in training make it impossible for the FIS to produce a suitable formulation, thus preventing the ANFIS training. 
Table 7 Final comparison of the behavior of the three tested artificial intelligence (AI) systems in estimation of hole diameters for the two alloys investigated

\begin{tabular}{|c|c|c|c|c|c|c|c|c|c|c|}
\hline \multicolumn{6}{|l|}{ Ti6Al4V alloy } & \multicolumn{5}{|c|}{ AA 2024-T3 alloy } \\
\hline \multirow[t]{3}{*}{ Parameter } & & & \multicolumn{3}{|c|}{ AI system } & \multicolumn{2}{|c|}{ Parameter } & \multicolumn{3}{|c|}{ AI system } \\
\hline & & & MLP & ANFIS & $\mathrm{RBF}$ & & & MLP & ANFIS & $\mathrm{RBF}$ \\
\hline & & & \multicolumn{5}{|c|}{ All input signals } & \multicolumn{3}{|c|}{ All input signals } \\
\hline \multicolumn{3}{|l|}{ Absolute error (mm) } & 0.008 & - & 0.0092 & \multicolumn{2}{|c|}{ Absolute error (mm) } & 0.0088 & - & 0.0176 \\
\hline \multicolumn{3}{|l|}{ Percent error (\%) } & 0.2 & - & 0.3 & \multicolumn{2}{|c|}{ Percent error $(\%)$} & 0.2 & - & - \\
\hline \multicolumn{3}{|l|}{ False positives $(\%)$} & 1.3 & - & 1.3 & \multicolumn{2}{|c|}{ False positives $(\%)$} & 9 & - & - \\
\hline \multicolumn{3}{|l|}{ False negatives $(\%)$} & 5.8 & - & 6.4 & \multicolumn{2}{|c|}{ False negatives (\%) } & 3.9 & - & - \\
\hline \multicolumn{3}{|l|}{ Hits $\leq 5 \mu \mathrm{m}(\%)$} & 52.5 & - & 43.5 & \multicolumn{2}{|c|}{ Hits $\leq 5 \mu \mathrm{m}(\%)$} & 42.5 & - & - \\
\hline \multicolumn{3}{|l|}{ Hits IT7 (\%) } & 35.5 & - & 40 & \multicolumn{2}{|c|}{ Hits IT7 (\%) } & 34 & - & - \\
\hline \multicolumn{3}{|l|}{ Hits IT10 (\%) } & 12 & - & 16.5 & Hits I' & $0(\%)$ & 23.5 & - & - \\
\hline & & & \multicolumn{3}{|c|}{ AI system } & & & \multicolumn{3}{|c|}{ AI system } \\
\hline & & & MLP & ANFIS & $\mathrm{RBF}$ & & & MLP & ANFIS & RBF \\
\hline & & & \multicolumn{5}{|c|}{$\mathrm{Z}$ component force $(\mathrm{FZ})$} & \multicolumn{3}{|c|}{$\mathrm{Z}$ component force (FZ) } \\
\hline \multicolumn{3}{|l|}{ Absolute error (mm) } & 0.0077 & 0.009 & 0.0102 & \multicolumn{2}{|c|}{ Absolute error (mm) } & 0.009 & 0.0102 & 0.0105 \\
\hline \multicolumn{3}{|l|}{ Percent error $(\%)$} & 02 & 0.3 & 0.2 & Perce & error $(\%)$ & 0.2 & 0.4 & 0.2 \\
\hline False positives $(\%)$ & & & 0.7 & 1.3 & 3 & False & sitives $(\%)$ & 6.4 & 5.8 & 7.7 \\
\hline False negatives $(\%)$ & & & 3.4 & 3.6 & 3.2 & False & gatives $(\%)$ & 18.5 & 12.9 & 11.2 \\
\hline Hits $\leq 5 \mu \mathrm{m}(\%)$ & & & 51 & 42 & 38 & Hits & $\mu \mathrm{m}(\%)$ & 43.5 & 44 & 48 \\
\hline Hits IT7 (\%) & & & 34.5 & 39.5 & 39.5 & Hits & $(\%)$ & 37 & 34 & 33 \\
\hline Hits IT10 $(\%)$ & & & 14.5 & 18 & 21.5 & Hits & $0(\%)$ & 19.5 & 21.5 & 19 \\
\hline & AI system & & & & & & AI system & & & \\
\hline & MLP & ANFIS & $\mathrm{RBF}$ & & & & MLP & ANFI & & $\mathrm{RBF}$ \\
\hline & Combined si & & & & & & Combined si & gnals & & \\
\hline & $\mathrm{AE}+\mathrm{ACC}$ & $\mathrm{FX}+\mathrm{FZ}$ & $\mathrm{FY}+\mathrm{ACC}$ & & & & $\mathrm{POW}+\mathrm{FZ}$ & $\mathrm{AE}+$ & $\mathrm{FZ}$ & $\overline{F Y+F Z}$ \\
\hline Absolute error (mm) & 0.0090 & 0.0102 & 0.0118 & Absc & lute error & $(\mathrm{mm})$ & 0.0085 & 0.010 & & 0.0175 \\
\hline Percent error (\%) & 0.2 & 0.35 & - & Perc & ent error (\% & & 0.2 & 0.4 & & - \\
\hline False positives $(\%)$ & 5.1 & 4.9 & - & False & positives & $(\%)$ & 5.8 & 4.5 & & - \\
\hline False negatives $(\%)$ & 2.8 & 1.9 & - & False & negatives & $(\%)$ & 7.5 & 17.2 & & - \\
\hline Hits $\leq 5 \mu \mathrm{m}(\%)$ & 44.5 & 35 & - & Hits & $\leq 5 \mu \mathrm{m}(\%$ & & 45.5 & 41 & & - \\
\hline Hits IT7 (\%) & 35 & 40 & - & Hits & IT7 (\%) & & 34 & 32 & & - \\
\hline Hits IT10 (\%) & 20.5 & 25 & - & Hits & IT10 (\%) & & 20.5 & 26.5 & & - \\
\hline
\end{tabular}

In the case of the RBF network, the failure to execute other tests is due to its poor generalization ability in the situations in question. This is attributed to the fact that this type of network is highly sensitive to the presentation of data and their relationship with the spread parameter.

Because of the spread tests in this study, which were performed in conjunction with the evaluation of the signals, were performed at moments other than the simulations to obtain further details, the parameter obtained in the tests did not correspond to the same results, except for the use of the $\mathrm{Z}$ component force input data.
RBF networks are similar to a MLP network with three layers, being one as an input, one as an output and one as a hidden layer. However, the two networks are different in the following aspects:

(a) A RBF network has one hidden layer, while a MLP may have one or more hidden layers.

(b) The compute nodes of an MLP, located in a hidden layer or an output layer, share a common neural model. Moreover, the computational nodes in the hidden layer of a RBF network are very different and they serve a 
different purpose from those of the network output layer.

(c) The hidden layer of an RBF network is nonlinear, while the output layer is linear. However, the hidden and output layers of a MLP network, when used as a pattern classifier, are all usually nonlinear.

(d) The argument of activation function in each hidden unit inside a RBF network calculates the Euclidean norm between the input vector and the center of that unit. Meanwhile, the activation function of each hidden layer in an MLP network calculates the inner product of the input vector by synaptic weight vector of that unit.

(e) MLP networks build global approximations from a non-linear input-output mapping. On the other hand, RBF networks using nonlinearities located with exponential decay build local approximations from nonlinear input-output mappings.

\section{Conclusions}

From this investigation on the experimental and numerical simulation tests conducted on drilling of sandwich plates of Ti6Al4V and AA 2024-T3 alloys with cemented carbide drills, the following conclusions can be drawn:

- ACC and FZ signals in general increased with the cutting speed when machining the Ti6Al4V alloy plate, irrespective of the feed rate employed, whereas the POW values decreased with increase in cutting speed. $\mathrm{AE}$ and POW parameters were more sensitive to variation in feed rate. Different pattern was observed for the diameter when using different cutting speed and feed rate combination;

- AE, ACC, POW and FZ signals increased with cutting speed when machining the AA 2024-T3 alloy. No significant influence of cutting speed and feed rate was observed on the diameter values. Values of most input parameters when machining the AA 2024-T3 were slightly higher than those for Ti6Al4V alloy;

- The MLP ANN outperformed the ANFIS and RBF networks, exhibiting great versatility with respect to variations in the data sets presented to it and maintaining low errors. This system proved reliable in the determination of valid results and most of its errors were below the precision the measuring instrument. A loss in performance was detected with elevation of the mean error in Ti6Al4V alloy, and this fact is consistent with the characteristic of the MLP ANN initially constituted on the filter base. Thus, in the presence of noisy signals, the ANN tends to ignore variations induced in the drilling process, producing a lower result than that found for the AA 2024-T3 alloy;
- The input with the $\mathrm{Z}$ component force was the only one able to present valid results for all the artificial intelligence systems analysed in this study because it yielded the best results in all the analysed criteria, with the exception of the quality control criterion in the systems configured for AA 2024-T3 alloy, which produced larger numbers of false results;

- The limitation of the number of inputs in ANFIS precludes the simultaneous use of more than four inputs. However, this network system was able to detect the poor machinability of the Ti6Al4V alloy;

- The RBF network exhibited great instability in response to the introduction of variations in the training set, which was evidenced by its low generalization ability;

- The potential to generate correct results in quality control was demonstrated by the low percentage of false results for the Ti6Al4V alloy. However, this was not confirmed for the AA 2024-T3 alloy;

- Regardless of the signals presented as inputs, the results analysed for the tolerances proved to be very reliable, since, among the valid results, a very small number of estimates was above the tolerance required by precision mechanics;

- $\mathrm{X}$ and $\mathrm{Y}$ components of machining forces, the $\mathrm{AE}+\mathrm{ACC}$ paired signals for the MLP ANN as well as the FY + ACC paired signals for the RBF network were marginally representative for the drilling of the Ti6Al4V alloy.

\section{References}

1. Kamen EW (1999) Industrial controls and manufacturing. Academic Press, San Diego

2. Huang SH, Zhang HC (1994) Artificial neural networks in manufacturing: concepts, applications, and perspectives. IEEE Trans Compon Packag Manuf Technol Part A 17(2):212-228

3. Matsushima M, Kawai N, Fujie H, Yasuda K (2010) Visual inspection of soldering joints by neural network with multi-angle view and principal component analysis. In: Shirase K, Aoyagi S (eds) Service robotics and mechatronics. Springer, London, pp 329-334

4. Aguiar PR, Paula WCF, Bianchi EC, Ulson JAC, Cruz CED (2010) Analysis of forecasting capabilities of ground surfaces valuation using artificial neural networks. J Braz Soc Mech Sci Eng 32(2):146-153

5. Cruz CED, Aguiar PR, Machado AR, Bianchi EC, Contrucci JG, Castro Neto F (2013) Monitoring in precision metal drilling process using multi-sensors and neural network. Int J Adv Manuf Technol 66:151-158

6. Rangwala S, Dornfeld. DA (1987) Integration of sensors via neural networks for detection of tool wear states intelligent and integrated manufacturing analysis and synthesis. In: Winter Meeting of the ASME, Boston, pp 109-120

7. Yang X, Kumehara H, Zhang W (2009) Back propagation wavelet neural network based prediction of drill wear from thrust force and cutting torque signals. Comput Inf Sci 2(3):75-86 
8. Li X, Tso SK (1999) Drill wear monitoring based on current signals. Wear 231(2):172-178

9. Abu-Mahfouz I (2003) Drilling wear detection and classification using vibration signals and artificial neural network. Int J Mach Tools Manuf 43(7):707-720

10. Kandilli I, Sonmez M, Ertunc HM, Cakir B (2007) Online monitoring of tool wear in drilling and milling by multi-sensor neural network fusion. In: International conference on mechatronics and automation. IEEE, pp 1388-1394

11. Youssef HA, El-Hofy H (2008) Machining technology: machine tools and operations. CRC Press, Boca Raton

12. Monostori L, Váncza J, Kumara SRT (2006) Agent-based systems for manufacturing. CIRP Ann Manuf Technol 55(2):697-720

13. Liang SY, Hecker RL, Landers RG (2004) Machining process monitoring and control: the state-of-the-art. J Manuf Sci Eng 126(2):297

14. Davim JP (2008) Machining: fundamentals and recent advances, 2nd edn. Springer, London

15. Haykin S (1999) Neural networks: a comprehensive foundation, 2nd edn. Pearson Prentice Hall, Delhi

16. Wilamowski BM, Irwin JD (2011) The industrial electronics handbook: intelligent systems. CRC Press, Boca Raton

17. Zadeh LA (1965) Fuzzy sets. Inf Control 8:338-353

18. Zadeh LA (1994) Fuzzy logic, neural networks, and soft computing. Commun ACM 37(3):77-84

19. Dote Y, Ovaska SJ (2001) Industrial applications of soft computing: a review. Proc IEEE 89(9):1243-1265

20. Chinnam RB, Ding J, May GS (2000) Intelligent quality controllers for on-line parameter design. IEEE Trans Semicond Manuf 13(4):481-491

21. Hermann G (2003) Application of neural network based sensor fusion in drill monitoring. In: 1st Slovakian-Hungarian joint simposium on applied machine intelligence. Budapest polytechnic, annals of conference, pp 1-14

22. Bustillo A, Correa M, Reñones A (2011) A virtual sensor for online fault detection of multitooth-tools. Sensors 11:2773-2795

23. Abellan-Nebot JV, Romero Subirón F (2009) A review of machining monitoring systems based on artificial intelligence process models. Int J Adv Manuf Technol 47(1-4):237-257

24. Bustillo A, Correa M (2012) Using artificial intelligence to predict surface roughness in deep drilling of steel components. J Intell Manuf 23(5):1893-1902
25. Ferreiro S, Sierra B, Irigoien I, Gorritxategi N (2011) Data mining for quality control: burr detection in the drilling process. Comput Ind Eng 60:801-810

26. Grzenda M, Bustillo A, Zawistowski P (2012) A soft computing system using intelligent imputation strategies for roughness prediction in deep drilling. J Intell Manuf 23:1733-1743

27. Wu X, Liu J (2009) A new early stopping algorithm for improving neural network generalization. In: Proceedings of the second international conference on intelligent computation technology and automation. IEEE, vol 1, pp 15-18

28. Brazilian Association of Technical Standards (1995) ABNT NBR 6158: System of limits and fits-Procedure. Brazilian Standard. Rio de Janeiro, 79 [IN PORTUGUESE: ASSOCIAÇÃO BRASILEIRA DE NORMAS TÉCNICAS-ABNT NBR 6158: Sistema de tolerâncias e ajustes]. Rio de Janeiro, 1995, p 79

29. Trent EM, Wright PK (2000) Metal cutting, 4th edn. Butterworth-Heinemann, Woburn, p 245

30. Bustillo A, Díez-Pastor JP, Quintana G, Osorio CG (2001) Avoiding neural network fine tuning by using ensemble learning: application to ball-end milling operations. Int J Adv Manuf Technol 57:521-532

31. Vaxevanidis NM, Kechagias JD, Fountas NA, Manolakos DE (2013) Three component cutting force system modelling and optimization in turning of AISI D6 tool steel using design of experiments and neural network. In: Proceedings of the World Congress on Engineering 2013 vol I, WCE 2013, London, UK

32. Noori-Khajavi A, Komanduri R (1993) On multisensor approach to drill wear monitoring. Ann CIRP 42(1):71-74

33. Patten JA, Jacob J, Bhattacharya B, Grevstad A, Fang N, Marsh ER (2007) Numerical simulations and cutting experiments on single point diamond machining of semiconductors and ceramics, semiconductor machining at the micro-nano scale, chapter 2 . Transw Res Netw Kerala India 2:1-36

34. Nabhani F (2001) Wear mechanisms of ultra-hard cutting tools materials. J Mater Process Technol 115:402-412

35. Da Silva RB, Da Silva MB, Sales WF, Ezugwu EO, Machado AR (2016) Advances in the turning of titanium alloys with carbide and superabrasive cutting tools. Adv Mater Res Trans Tech Publ Switz 1135:234-254 